TRANSACTIONS OF THE

AMERICAN MATHEMATICAL SOCIETY

Volume 361, Number 7, July 2009, Pages 3435-3462

S 0002-9947(09)04849-1

Article electronically published on February 23, 2009

\title{
AUTOMORPHISMS OF THE LATTICE OF EQUATIONAL THEORIES OF COMMUTATIVE SEMIGROUPS
}

\author{
MARIUSZ GRECH
}

\begin{abstract}
In this paper we complete the study of the first-order definability in the lattice of equational theories of commutative semigroups started by A. Kisielewicz in [Trans. Amer. Math. Soc. 356 (2004), 3483-3504]. We describe the group of automorphisms of this lattice and characterize firstorder definable theories, thus solving the problems posed by R. McKenzie and A. Kisielewicz.
\end{abstract}

\section{INTRODUCTION}

In 13, 11] A. Tarski and R. McKenzie raised problems of first-order definability in the lattices of equational theories. In a series of papers [3, 4, 5, 6] Ježek has solved the most general problems in this area considering the case of lattices of equational theories of a given type.

In [7. Ježek and McKenzie have studied the first-order definability in the lattice of equational theories of semigroups. They have proved that in this lattice many interesting sets of theories are definable (e.g. the finitely axiomatizable theories, the locally finite theories, and the theories of finite semigroups). Moreover, they have proved that each individual finitely axiomatizable locally finite theory is definable up to duality (i.e. up to inverting the order of occurrence of letters in defining equations). They conjecture that the assumption on local finiteness in the last quoted result may be omitted and that, in consequence, the lattice in question has no nontrivial automorphisms, but these problems still remain open.

One of the reasons that the problems above seem very hard is that we have very little detailed knowledge about the structure of the lattice of equational theories of semigroups. In contrast, we have very good knowledge about the structure of the lattice $L(\mathrm{Com})$ of equational theories of commutative semigroups [8]. Nevertheless, this lattice seemed large and complicated enough so that one could believe that every individual theory is definable in it and that it has no nontrivial automorphisms. In particular, the fact that, on the one hand, $L(\mathrm{Com})$ contains all finite lattices and, on the other hand, each theory in $L(\mathrm{Com})$ is finitely axiomatizable, made this conjecture quite plausible.

So, it came as a surprise when Kisielewicz [9] (using the description in [8]) discovered that there exist nontrivial automorphisms of $L(\mathrm{Com})$. A generalization of Kisielewicz's example by McKenzie has even shown that the group of automorphisms of $L(\mathrm{Com})$ contains a Boolean group of order continuum. Although

Received by the editors May 15, 2006.

2000 Mathematics Subject Classification. Primary 03C07; Secondary 03C05, 08B15. 
many sets of theories and many individual theories were shown in [9] to be firstorder-definable in $L(\mathrm{Com})$, a few interesting questions remained open: What is the group of automorphisms of $L(\mathrm{Com})$ ? Does it have any elements of order greater than 2? Are there any automorphisms of a different type than those constructed by Kisielewicz and McKenzie based on exchanging equations? Which theories are definable and which are not? This paper offers the full answer to all these questions, thus solving the problems posed in 9 and completing the work started there.

The main idea that allowed us to get these results was focusing strongly on joinirreducible theories. (In 9], Kisielewicz worked mainly with one-based theories.) The property of being one-based does not seem to have much to do with automorphisms of the lattice and, in particular, it was not even clear whether or not this property was preserved by automorphisms. We chose to restrict ourselves to the join-irreducible theories, which are fully characterized in [1]. They are obviously definable as a set, and the image of a join-irreducible theory under any automorphism is join-irreducible. We prove that each theory, whose definability is not established by methods of [9], is a finite join of join-irreducible theories. This crucial result proved in Section 4 makes a breakthrough possible. Of course, we make heavy use of the machinery worked out in [8, 9, 2, and the results on join-irreducible theories in 1 .

The terminology and results from Kisielewicz' fundamental papers 8 and 9, which we will apply repeatedly in this paper, are summarized for the reader's convenience in Section 2. To achieve our aim, we need detailed knowledge about the structure of certain permutation groups associated naturally with equations. These groups and the facts we need are introduced in Section 3. In Section 4 we reduce our problem to join-irreducible theories. After these preparations, in Section 5, we prove that every theory generated by an equation of the form $(\mathbf{a}, p(\mathbf{a}))$ is definable. This result and other lemmas also allow us to prove that most of the one-based theories are definable, and there remain only very special equations to consider among which we find and characterize those generating nondefinable theories. This last part is fairly technical and requires considering a number of different cases. An explicit description of the set of exceptional equations makes it possible to give a full description both of the set of nondefinable theories and of the group of automorphisms of $L(\mathrm{Com})$. These results are presented in the last section. In particular, it also follows from our results that being one-based is a property of theories which $i s$ preserved by automorphisms of the lattice.

\section{Preliminaries}

We assume that the reader has basic knowledge in the areas of first-order logic, equational logic and lattice theory. In this section we recall the terminology and facts proved in [8, 1, 9, which are used extensively throughout the paper. We start with the description of the lattice $L(C o m)$ given in 8 .

Let $\Gamma$ be the set of all finite sequences $\left(a_{1}, \ldots, a_{n}\right)$ of nonnegative integers such that at least one $a_{i} \neq 0$. For a sequence $\mathbf{a}=\left(a_{1}, \ldots a_{n}\right) \in \Gamma$ and a permutation of the indices $f \in S_{n}$, we put $f(\mathbf{a})=\left(a_{f(1)}, \ldots, a_{f(n)}\right)$. Moreover, for every $\gamma \geq$ 0 we denote $h_{\gamma}(\mathbf{a})=\left(a_{1}, \ldots, a_{n}, \gamma\right)$, and for $1 \leq i, j \leq n, i \neq j$, we denote $g_{i j}(\mathbf{a})=\left(a_{1}, \ldots, a_{i}+a_{j}, \ldots, a_{n}\right)$, where the latter is the sequence obtained from $\mathbf{a}$ by replacing $a_{i}$ by $a_{i}+a_{j}$, and deleting $a_{j}$. 
For sequences $\mathbf{a}, \mathbf{b} \in \Gamma$, we define $\mathbf{a} \leq \mathbf{b}$ if and only if there exists a function $f$ which is a composition of permutations, the functions $h_{0}, h_{1}$ and some of the $g_{i j}$, such that $f(\mathbf{a})=\mathbf{b}$.

It is proved in 8 that $(\Gamma, \leq)$ is a well-quasi-ordered set, which means, in particular, that there are no infinite descending chains in $\Gamma$ and every (order) filter $J$ in $\Gamma$ is finitely generated. A filter generated by the sequences $\mathbf{a}_{1}, \ldots, \mathbf{a}_{n}$ is denoted by $J=\left[\mathbf{a}_{1}, \ldots, \mathbf{a}_{n}\right]$.

A commutative semigroup equation of the form

$$
x_{1}^{a_{1}} \cdots x_{n}^{a_{n}}=x_{1}^{b_{1}} \cdots x_{n}^{b_{n}}
$$

will be identified with the pair $(\mathbf{a}, \mathbf{b})$ of sequences $\mathbf{a}=\left(a_{1}, \ldots, a_{n}\right)$ and $\mathbf{b}=$ $\left(b_{1}, \ldots, b_{n}\right)$, and denoted briefly as $(\mathbf{a}, \mathbf{b})$. In using this notation, we always assume that for all $1 \leq i \leq n$ we have $a_{i}+b_{i}>0$. An equation is nontrivial if $a_{i} \neq b_{i}$ for some $i$. It is regular if all $a_{i}, b_{i}>0$.

An equational theory of commutative semigroups (or briefly, a theory) is a nonempty set of equations closed on taking logical consequences (which apart from substitutions corresponds to applying $g_{i j}, h_{1}$ and permutations to pairs $(\mathbf{a}, \mathbf{b})$, and using transitivity). The least theory, consisting of the trivial equations $(\mathbf{a}, \mathbf{a})$, is called the trivial theory.

For a nonempty filter $J$ in $\Gamma$, and integers $m \geq 0, r>0$, we define $E(J, m, r)$ to be the set consisting of those pairs $(\mathbf{a}, \mathbf{b})$ with $\mathbf{a}, \mathbf{b} \in J$ that satisfy the following conditions:

$\left(C^{r}\right)$ for every $i, a_{i} \equiv b_{i}(\bmod r)$,

$\left(C_{m}\right)$ for every $i$, if $a_{i} \neq b_{i}$, then $a_{i}, b_{i} \geq m$,

together with all the trivial equations $(\mathbf{a}, \mathbf{a})$. It is known that all such sets are equational theories, referred to as Schwabauer theories.

In order to describe more complex theories we consider some conditions for equations. Let $\Gamma_{+}$denote the set of all finite nonvoid sequences of positive integers. Let $\pi$ be an equivalence relation on some subset of $\Gamma_{+}$. Let $m \geq 0, r>0$ be integers as above. Then let $(\mathbf{a}, \mathbf{b})$ be any equation where $\mathbf{a}=\left(a_{1}, \ldots, a_{n}\right), \mathbf{b}=\left(b_{1}, \ldots, b_{n}\right) \in$ $\Gamma_{+}$.

$(\pi 1)$ The conditions $\left(C^{r}\right),\left(C_{m}\right)$ hold,

$(\pi 2)$ for every permutation $f \in S_{n},(f(\mathbf{a}), f(\mathbf{b})) \in \pi$,

$(\pi 3)$ for every $i$ such that $a_{i} \neq b_{i}$, and $\gamma=\min \left(a_{i}, b_{i}\right)$, both $h_{\gamma}(\mathbf{a}), h_{\gamma}(\mathbf{b}) \in J$,

$(\pi 4)$ if $f$ is one of the operations $g_{i j}$ or $h_{1}$, then either $(f(\mathbf{a}), f(\mathbf{b})) \in \pi$ or both $f(\mathbf{a}), f(\mathbf{b}) \in J$.

Now let $m \geq 0, r>0$ be integers, $J$ be a nonempty filter contained in $[(m)]$ and $\pi$ be an equivalence relation on the set $\Gamma_{+} \backslash J$ of those sequences of positive integers that are not in $J$. If every block of $\pi$ contains sequences of one fixed length and for all pairs $(\mathbf{a}, \mathbf{b}) \in \pi$ the conditions $(\pi 1)-(\pi 4)$ are satisfied, then $\pi$ is called a remainder of the type $(J, m, r)$. For such a remainder we define $E(J, m, r, \pi)=E(J, m, r) \cup \pi$. Then:

Theorem 2.1 (8]). Every set $E(J, m, r, \pi)$ defined above is an equational theory, and every nontrivial equational theory of commutative semigroups is of this form.

Note that it follows from $(\pi 4)$ that if $(\mathbf{a}, \mathbf{b}) \in \pi$, then the sequences $\mathbf{a}, \mathbf{b}$ are equivalent or incomparable. In particular, all blocks in the remainders are finite. It also follows from $(\pi 3)$ that if we substitute $y z$ for a variable $x$ which has different 
occurrences in $\mathbf{a}$ and $\mathbf{b}$, then we get an equation $\left(\mathbf{a}^{\prime}, \mathbf{b}^{\prime}\right)$ with both $\mathbf{a}^{\prime}, \mathbf{b}^{\prime} \in J$. This can be viewed as a major advantage of Kisielewicz' characterization that substitutions are effectively removed from the considerations of equational logic. Thus, every equational theory consists of two parts: $E(J, m, r)$, which is described in purely arithmetical terms, and the remainder $\pi$, where logical consequences are restricted to applying operations $g_{i j}, h_{1}$ and permutations to pairs (a,b), and using transitivity. These parts are disjoint (modulo trivial equations), and the remainder may be viewed, in view of $(\pi 4)$, as lying below the filter. Schwabauer theories are those whose remainder $\pi$ is trivial, i.e. consists of equations of the type $(\mathbf{a}, \mathbf{a})$.

The part $E(J, m, r)$ is completely determined by generators of $J$, the relation $\leq$ on $\Gamma$, and conditions $\left(C^{r}\right)$ and $\left(C_{m}\right)$. It is a nontrivial task to determine $J$ knowing a base for a theory $E$. For an algorithm and examples the reader is referred to 8 . We shall not use it in this paper. It may be however illuminating to know that a sequence a corresponding to a word $w$ belongs to $J$ if and only if an equation of the form $u w=w$ belongs to $E$ for some nonempty word $u$. For $u$ one may take, for example, $x^{c+r}$, providing $x^{c}$ occurs in $w$, and $c \geq m$.

We now consider in more detail the structure of the remainders. First we note that they are closed under the action of the symmetric group $S_{n}$ for every $n$ : If $n$ is the length of the sequences in a block $B$ of a remainder $\pi$, then $p(B)$ is also a block of $\pi$ for any $p \in S_{n}$. Further, denote by $G_{B}$ the setwise stabilizer of $B$ in $S_{n}$. Then $B$ is generated by the sequences forming a maximal antichain in $B$ as follows.

Lemma 2.2 ([1]). For every block $B$ of a remainder $\pi$, if $A$ is a maximal antichain contained in $B$, then $B=G_{B} A=\left\{g(\mathbf{a}): g \in G_{B}, \mathbf{a} \in A\right\}$.

We shall use a quasi-ordering on the blocks of $\pi$. Let $B$ and $C$ be blocks in $\pi$. We define $B \leq C$ if and only if $f(B) \subseteq C$ for some $f$ generated by $g_{i j}, h_{1}$ and permutations. We note that under this quasi-ordering, $B$ and $C$ are equivalent if and only if $p(B)=C$ for some permutation $p$ (see [1]).

According to Lemma 2.2 the blocks in $\pi$ are of the form $B=G A$, where $G$ is a subgroup of $S_{n}$ and $A$ is an antichain of sequences of length $n$. (By "antichain", we mean with respect to the quasi-ordering on sequences of integers already introduced above.) By $[B]$ or $[G A]$ we denote the orbit of $B$ under the action of $S_{n}$, consisting of all sets of the form $g(G A), g \in S_{n}$. Note that this is exactly the equivalence class of $B$ in the quasi-ordering defined above (i.e. consists of all blocks equivalent to $B$ ). We say that a theory $E$ is generated by the block $B=G A$ if it is generated by the set of equations $\{(\mathbf{a}, \mathbf{b}): \mathbf{a}, \mathbf{b} \in B\}$. Observe that in this case every set from the class $[G A]$ is a minimal nontrivial block in the remainder of $E$ and that the block $B$ is unique up to equivalence.

Finally, we turn to the matters of definability. Recall that a subset $S$ of a lattice $L$ is first-order definable in $L$ if there exists a formula $\Phi(x)$ in the first-order language of the lattice such that $\Phi(x)$ holds if and only if $x \in S$. An element $x_{0} \in L$ is definable if the set $\left\{x_{0}\right\}$ is definable. Accordingly, we speak of definable relations between elements of $L$ and speak of definable properties of the elements in $L$. In this paper, we are concerned entirely with the lattice $L=L(\mathrm{Com})$ of equational theories of commutative semigroups. We now introduce some needed results from A. Kisielewicz [9] regarding definability in this lattice.

Theorem 2.3 ([9, Theorem 4.3]). Every Schwabauer theory is definable. Also the set of Schwabauer theories is definable. 
For a theory $E=E(J, m, r, \pi)$, define $J(E)=J, m(E)=m$ and $r(E)=r$. Then we have

Theorem 2.4 ([9, Proposition 4.2 and the proof of Theorem 4.3]). Given integers $m \geq 0, r>0$ and a filter $J$ over $\Gamma$ the following sets are definable:

(a) the set of theories $E$, with $J(E)=J$,

(b) the set of theories $E$, with $m(E)=m$,

(c) the set of theories $E$, with $r(E)=r$.

We will focus our attention on the definability of join-irreducible theories. The following will be very useful to this aim.

Theorem 2.5 ([9, Theorem 5.1 and Theorem 5.4]). Given two incomparable sequences $\mathbf{a}, \mathbf{b}$ of positive integers with length $n$, the following sets are definable:

(a) the set of theories generated by a set of equations of the form $(\mathbf{a}, p(\mathbf{a}))$, $p \in S_{n}$,

(b) the set of theories generated by a set of equations of the form $(\mathbf{a}, p(\mathbf{b}))$, $p \in S_{n}$.

We note that it does not follow from these claims that the set of theories generated by a single equation of the form $(\mathbf{a}, p(\mathbf{a}))($ or $(\mathbf{a}, p(\mathbf{b})))$ is definable. Although it may be easily seen (using e.g. Lemma 3.1 below) that a one-based theory generated by a set of equations of the form $(\mathbf{a}, p(\mathbf{a}))$ is generated by one of these equations, we cannot make use of this, because we do not know whether the set of one-based theories is definable. The situation is different for join-irreducible theories, and we get a suitable corollary in the next section.

We will also use the following result concerning definability of individual theories generated by the so called 'balanced' equations.

Theorem 2.6 ([9, Theorem 6.5]). Let $\mathbf{a}, \mathbf{b}$ be incomparable sequences of the same length such that for all $i, j, u, w$, sequences $g_{i j}(\mathbf{a})$ and $g_{u w}(\mathbf{b})$ are incomparable. Then the theory generated by equation $(\mathbf{a}, \mathbf{b})$ is definable.

\section{EQUATIONS AND GROUPS OF PERMUTATIONS}

Let us recall that by virtue of [12] every equational theory of commutative semigroups is finitely generated. This means that considering definability of individual theories we may concentrate on one-based theories. We shall write $E(\mathbf{a}, \mathbf{b})$ to denote the theory generated by an equation $(\mathbf{a}, \mathbf{b})$.

By results of [8] every theory generated by an irregular equation, as well as every theory generated by an equation $(\mathbf{a}, \mathbf{b})$ with comparable but nonequivalent sequences, is a Schwabauer theory. By Theorem 2.3 these theories are definable. Thus we may restrict to theories $E(\mathbf{a}, \mathbf{b})$, where $\mathbf{a}, \mathbf{b}$ are equivalent or incomparable sequences of positive integers. Henceforth, all sequences mentioned are assumed to consist of positive integers, and all theories mentioned are assumed to be regular, unless otherwise stated. For such theories, we have

Lemma 3.1. Let $\mathbf{a}, \mathbf{b}$ be different sequences of positive integers which are equivalent or incomparable. Then the theory $E(\mathbf{a}, \mathbf{b})$ generated by equation $(\mathbf{a}, \mathbf{b})$ has a nontrivial remainder $\pi$ and is generated by a minimal block $B$ of $\pi$ containing a and $\mathbf{b}$. Moreover, $B=G_{B}\{\mathbf{a}, \mathbf{b}\}$, where $G_{B}$ is the setwise stabilizer of $B$, and if $\mathbf{a}$ and $\mathbf{b}$ are equivalent, then $B=G_{B}\{\mathbf{a}\}$. 
Proof. For $\mathbf{a}$ and $\mathbf{b}$ equivalent this easily follows from the result in Section 2. Then $\mathbf{a}=p(\mathbf{a})$ for some permutation $p \in S_{n}$, and each consequence $(\mathbf{c}, \mathbf{d})$ of $(\mathbf{a}, p(\mathbf{a}))$ is obtained either by applying a permutation or both $\mathbf{c}, \mathbf{d}$ are strictly larger in the ordering described in Section 2. It follows that there is no equation $(\mathbf{a}, \mathbf{b})$ in $E(\mathbf{a}, p(\mathbf{a}))$ with $\mathbf{b}$ strictly larger than $\mathbf{a}$, and therefore $(\mathbf{a}, \mathbf{b})$ is not in the Schwabauer part of the theory. Hence, the remainder is nontrivial. Since $E(\mathbf{a}, p(\mathbf{a}))$ is generated by $(\mathbf{a}, p(\mathbf{a}))$, the latter has to be in the minimal block $B$, unique up to equivalence (cf. also the remarks following Lemma 2.2). Moreover, it is clear that $B$ consists of permutations of the sequence $\mathbf{a}$ in this case, and therefore $B=G_{B}\{\mathbf{a}\}$ (i.e. the maximal antichain mentioned in Lemma 2.2 is one-element).

For $\mathbf{a}$ and $\mathbf{b}$ incomparable the argument is similar. We observe that for any nonpermutation consequence $(\mathbf{c}, \mathbf{d})$ of $(\mathbf{a}, \mathbf{b})$, either $\mathbf{c}$ is strictly larger than a and strictly larger or incomparable with $\mathbf{b}$, or else $\mathbf{c}$ is strictly larger than $\mathbf{b}$ and strictly larger or incomparable with $\mathbf{a}$ (otherwise $\mathbf{a}$ and $\mathbf{b}$ would be comparable). Hence, as before, $(\mathbf{a}, \mathbf{b})$ belongs to a minimal block $B$ in a nontrivial remainder. In this case there are two-element maximal antichains in $B$, and $B=G_{B}\{\mathbf{a}, \mathbf{b}\}$.

It easily follows that for fixed sequences $\mathbf{a}, \mathbf{b}$ as in Lemma 3.1, a one-based theory generated by a set of equations of the form $(\mathbf{a}, p(\mathbf{b}))$ is generated by one such equation. Taking into account the facts that join-irreducible theories are one-based and the set of join-irreducible theories is definable, we obtain:

Corollary 3.2. Given two incomparable sequences $\mathbf{a}, \mathbf{b}$ of positive integers with length $n$, the following sets are definable:

(a) the set of join-irreducible theories generated by a single equation of the form $(\mathbf{a}, p(\mathbf{a})), p \in S_{n}$;

(b) the set of join-irreducible theories generated by a single equation of the form $(\mathbf{a}, p(\mathbf{b})), p \in S_{n}$.

Now we wish to describe the structure of $G_{B}$ in Lemma 3.1. Although generally the stabilizer $G_{B}$ of a block $B$ may be an arbitrary permutation group, in the considered cases the structure of $G_{B}$ is fairly simple.

First, let us establish some notation concerning permutation groups. By $S_{X}$ we denote the symmetry group acting on the set $X$; we write $S_{n}$ for $X=\{1,2, \ldots, n\}$. Given two sets of permutations $P$ and $Q$, by $\langle P, Q\rangle$ we denote the group generated by the union of $P$ and $Q$. For two groups of permutations $(G, X)$ and $(H, Y)$ acting on disjoint sets $X$ and $Y$, respectively, $G \times H=(G \times H, X \cup Y)$ denotes the direct product (sometimes called the direct sum) of permutation groups, i.e. the abstract product $G \times H$ acting on the set $X \cup Y$ in the natural way: $(g, h)(z)=g(z)$ or $h(z)$ depending on whether $z \in X$ or $Y$.

When we are discussing $S_{X}$, and $I$ is a subset of $X$, we shall sometimes write (by an abuse of notation) $S_{I}$ for the subgroup of $S_{X}$ consisting of those permutations of $X$ that leave the elements of $X \backslash I$ pointwise fixed. We now recall an easy fact, which we shall use tacitly many times. (See for instance [10.)

Lemma 3.3. Let $p$ be a permutation of the set $X=\{1, \ldots, n\}$, and $I \subseteq X$. If there exist $a, b \in I$ such that $p(a) \in I$ and $p(b) \notin I$, then $\left\langle p, S_{I}\right\rangle \supseteq S_{J}$, where $J=I \cup\{p(b)\}$.

Definition 3.4. Given a sequence $\mathbf{a}=\left(a_{1}, \ldots, a_{n}\right)$, by $G(\mathbf{a})$ we denote the symmetry group of $\mathbf{a}$, that is, the set of permutations $\sigma \in S_{n}$ satisfying $a_{\sigma(i)}=a_{i}$ for all 
$i \leq n$. For an equation $(\mathbf{a}, \mathbf{b})$ by $G(\mathbf{a}, \mathbf{b})$ we denote the group of those permutation $q \in S_{n}$ for which $(\mathbf{a}, q(\mathbf{b})) \in E(\mathbf{a}, \mathbf{b})$.

It should be clear that every $G(\mathbf{a})$ is the direct product of the form $S_{X_{1}} \times \cdots \times S_{X_{k}}$, where $\left\{X_{1}, \ldots, X_{k}\right\}$ is a partition of the set $X=\{1,2, \ldots, n\}$. Since $E(\mathbf{a}, \mathbf{b})$ is not a Schwabauer theory (by our working assumption), then it follows that $G(\mathbf{a}, \mathbf{b})$ coincides with the setwise stabilizer $G_{B}$ of the minimal block $B$ in Lemma 3.1 .

In the case when $\mathbf{a}, \mathbf{b}$ are incomparable, elementary reasoning on consequences obtained by applying permutations to the equation $(\mathbf{a}, \mathbf{b})$ yields that $G(\mathbf{a}, \mathbf{b})=$ $\langle G(\mathbf{a}), G(\mathbf{b})\rangle$. The latter by Lemma 3.3 is of the form $S_{Y_{1}} \times \cdots \times S_{Y_{k}}$, where $\left\{Y_{1}, \ldots, Y_{k}\right\}$ is a partition of the set $X=\{1,2, \ldots, n\}$. The sets $Y_{i}$ are in fact the orbits of $G(\mathbf{a}, \mathbf{b})$.

If $\mathbf{a}$ is equivalent with $\mathbf{b}$, then $\mathbf{b}=p(\mathbf{a})$ for some permutation $p \in S_{n}$, and the situation is only a little bit more complicated. As it is easy to see, the block $B$ generating $E(\mathbf{a}, p(\mathbf{a}))$ consists of those permutations of the sequence a that can be obtained by combining permutations of $G(\mathbf{a})$ with permutation $p$. Thus $G(\mathbf{a}, p(\mathbf{a}))=\langle p, G(\mathbf{a})\rangle$, and using Lemma 3.3 we see that if $\left\{Y_{1}, \ldots, Y_{k}\right\}$ is the least partition preserved by permutation $p$ and containing the orbits of $G(\mathbf{a})$ (as a subpartition), then $p$ has an induced action on $\left\{Y_{1}, \ldots, Y_{k}\right\}$, and $G(\mathbf{a}, p(\mathbf{a}))$ is generated by the product $S_{Y_{1}} \times \cdots \times S_{Y_{k}}$ and the permutation $p$ permuting the whole blocks $Y_{i}$. Let us summarize this in the following

\section{Lemma 3.5.}

(1) Suppose that $\mathbf{a}, \mathbf{b}$ are incomparable sequences of length $n$. Then $G(\mathbf{a}, \mathbf{b})=$ $\langle G(\mathbf{a}), G(\mathbf{b})\rangle=S_{Y_{1}} \times \ldots \times S_{Y_{k}}$, where $Y_{1}, \ldots, Y_{k}$ are blocks of the equivalence relation determined by the orbit decompositions of $X=\{1,2, \ldots, n\}$ with respect to $G(\mathbf{a})$ and with respect to $G(\mathbf{b})$. The $Y_{i}$ are, of course, also orbits of $G(\mathbf{a}, \mathbf{b})$.

(2) Let $p$ be a nontrivial permutation. Then $G(\mathbf{a}, p(\mathbf{a}))$ is the group generated by $S_{Y_{1}} \times \cdots \times S_{Y_{k}}$ and permutation $p$, where $\left\{Y_{1}, \ldots, Y_{k}\right\}$ is the least partition of $X$ preserved by permutation $p$ and containing the orbits of $G(\mathbf{a})$.

\section{REDUCTION to JOIN-IRREDUCIBLE THEORIES}

In 9 the problems of definability have been reduced to one-based theories. However, this approach does not work in general, because the property of being onebased is not easily seen to be preserved by automorphisms. Therefore we focus on join-irreducible theories. They are certainly much more natural in considering problems of definability, but generally it is not true that every theory in $L(\mathrm{Com})$ is a join of a finite set of such theories. Nevertheless we show in this section that taking into account the results already established in 9] we can now restrict ourselves to join-irreducible theories.

Our main reduction result is the following.

Theorem 4.1. Every theory $E \in L(C o m)$ generated by regular equations with incomparable or equivalent sequences is a join of a finite set of join-irreducible theories.

Proof. In view of Perkins' result [12] we may assume that $E$ is a join of a finite set of equational theories, each generated by a single regular equation with incomparable or equivalent sequences. Let $(\mathbf{a}, \mathbf{b})$ be one of these equations, and let 
$E_{1}=E(\mathbf{a}, \mathbf{b})$ be the theory generated by $(\mathbf{a}, \mathbf{b})$. By Lemma 3.1. $E_{1}$ can also be viewed as generated by a minimal block $B$ in the remainder with $B=G_{B}\{\mathbf{a}, \mathbf{b}\}$.

Assume that $E_{1}$ is not join-irreducible. Then, since (by [12]) there are no infinite ascending chains in $L(C o m), E_{1}$ is a join of two dual covers. Let $E_{2}$ and $E_{3}$ be two different dual covers of $E(\mathbf{a}, \mathbf{b})$, and let $n$ be the common length of the sequences a and $\mathbf{b}$. Since every theory is finitely generated, we can assume that $E_{2}$ is generated by a set of nontrivial equations $S_{2}=\left\{\left(\mathbf{a}_{1}, \mathbf{b}_{1}\right), \ldots,\left(\mathbf{a}_{t}, \mathbf{b}_{t}\right)\right\}$ and that $E_{3}$ is generated by a set of nontrivial equations $S_{3}=\left\{\left(\mathbf{c}_{1}, \mathbf{d}_{1}\right), \ldots,\left(\mathbf{c}_{s}, \mathbf{d}_{s}\right)\right.$. Put $S_{2}^{\prime}=\left\{\left(\mathbf{a}_{i}, \mathbf{b}_{i}\right) \in\right.$ $\left.S_{2}: \mathbf{a}_{i}, \mathbf{b}_{i} \in S_{n}\{\mathbf{a}, \mathbf{b}\}\right\}$ and, analogously, $S_{3}^{\prime}=\left\{\left(\mathbf{c}_{j}, \mathbf{d}_{j}\right) \in S_{3}: \mathbf{c}_{j}, \mathbf{d}_{j} \in S_{n}\{\mathbf{a}, \mathbf{b}\}\right\}$. Let $E_{2}^{\prime}$ and $E_{3}^{\prime}$ be the theories generated by $S_{2}^{\prime}$ and $S_{3}^{\prime}$, respectively. We show that $E_{1}$ is a join of $E_{2}^{\prime}$ and $E_{3}^{\prime}$.

First note that since $S_{2} \cup S_{3}$ is a subset of $E_{1}$ and the latter is generated by the minimal block $B=G_{B}\{\mathbf{a}, \mathbf{b}\}$, for each equation $(\mathbf{c}, \mathbf{d}) \in S_{2} \cup S_{3}$, either $\mathbf{c}, \mathbf{d} \in S_{n}\{\mathbf{a}, \mathbf{b}\}$ or each of the sequences $\mathbf{c}$ and $\mathbf{d}$ is strictly greater than one of the sequences $\mathbf{a}, \mathbf{b}$. Now, we show that in order to infer the equation $(\mathbf{a}, \mathbf{b})$ it is enough to use only the set $S_{2}^{\prime} \cup S_{3}^{\prime}$.

Indeed, $(\mathbf{a}, \mathbf{b})$ can be obtained from $S_{2} \cup S_{3}$ by applying operations $g_{i j}, h_{1}$, permutations and transitivity. Applying permutation or transitivity to the set of equation of the form $(\mathbf{c}, \mathbf{d})$, where $\mathbf{c}, \mathbf{d} \in S_{n}\{\mathbf{a}, \mathbf{b}\}$, we can obtain only the equations of the same form. Applying the operations $g_{i j}, h_{1}$ to the set of the equations of this form, we obtain only the equations $(\mathbf{c}, \mathbf{d})$, where each of the sequences $\mathbf{c}, \mathbf{d}$ is larger than at least one of $\mathbf{a}, \mathbf{b}$. The same can be obtained when we apply $g_{i j}, h_{1}$, permutation or transitivity to the set of the set of equations of the later form. We cannot apply transitivity to two equations of different forms. Hence, to obtain $(\mathbf{a}, \mathbf{b})$ we have to apply only permutations and transitivity, and we use only the equations from the set $S_{2}^{\prime} \cup S_{3}^{\prime}$.

It follows that $E_{1}$ is a join of the theories $E_{2}^{\prime}$ and $E_{3}^{\prime}$. Consequently, $E_{1}$ is a join of strictly smaller one-based theories of the form $E(\mathbf{c}, \mathbf{d})$, where $\mathbf{c}, \mathbf{d} \in S_{n}\{\mathbf{a}, \mathbf{b}\}$. If some of those theories are again join-reducible, then we can apply the same procedure to this theory. Since there is only a finite number of two element subsets of the set $S_{n}\{\mathbf{a}, \mathbf{b}\}$, this procedure will finish after a finite number of steps. As a result, we obtain a finite set of join-irreducible theories of this same form whose join is $E_{1}$.

The proof above has been suggested by an anonymous referee. Our original proof was based on the description of the covering relation given in [2. Theorem 2.2] and [1, Theorem 3.1]. While one can see that the result follows easily from this very description, the present proof is certainly more elementary.

We will also need the following corollary, which follows immediately from the method used in the proof above.

Corollary 4.2. If $E(\mathbf{a}, \mathbf{b})$ is a theory generated by a single regular equation with equivalent or incomparable sequences $\mathbf{a}$ and $\mathbf{b}$, then $E(\mathbf{a}, \mathbf{b})$ is a join of a finite number of join-irreducible theories generated by single equations involving only permutations of sequences $\mathbf{a}$ and $\mathbf{b}$.

Since every irregular equation or an equation with comparable sequences defines a Schwabauer theory, in general, we get the following.

Corollary 4.3. Every theory of $L(C o m)$ is a join of a finite set of Schwabauer theories and join-irreducible theories. 
Since by Theorem 2.3. under every automorphism of $L(C o m)$ Schwabauer theories remain fixed, we see that every automorphism of $L(\mathrm{Com})$ is determined by its action on join-irreducible theories. The image of a join-irreducible theory under any automorphism is join-irreducible. Moreover, each join-irreducible theory $E$ is generated by a single regular equation $(\mathbf{a}, \mathbf{b})$ with $\mathbf{a}, \mathbf{b}$ equivalent or incomparable (since Schwabauer theories are all join reducible; cf. 2, Theorem 2.2]). It follows by Corollary 3.2 that the image $\Phi(E)$ under automorphism $\Phi$ is generated by one equation $(\mathbf{a}, p(\mathbf{b}))$. Thus we have

Corollary 4.4. Every automorphism $\Phi$ of the lattice $L(C o m)$ is determined by its action on join-irreducible theories. The image $\Phi(E)$ of a join-irreducible theory $E$ generated by an equation $(\mathbf{a}, \mathbf{b})$ is a join-irreducible theory generated by an equation $(\mathbf{a}, p(\mathbf{b}))$, for some permutation $p$.

Thus, we have two types of join-irreducible theories: Those generated by a single equation $(\mathbf{a}, \mathbf{b})$ with incomparable sequences, and those generated by a single equation of the form $(\mathbf{a}, p(\mathbf{a}))$. We first prove that for the latter type each theory is individually definable.

\section{EquATIOns With EQUivalent SEQUENCES}

In this section we prove that every join-irreducible theory generated by a single equation of the form $(\mathbf{a}, p(\mathbf{a}))$ is definable in $L(C o m)$. The following technical lemma will play a central role in many of our arguments.

Lemma 5.1. Let $\mathbf{a}=\left(a_{1}, \ldots, a_{n}\right), p \in S_{n}$, and $E=E(\mathbf{a}, p(\mathbf{a}))$. Let $i \neq j, s \neq t$ be fixed. Then there exists $\sigma \in G(\mathbf{a}, p(\mathbf{a}))$ such that $\{\sigma(i), \sigma(j)\}=\{s, t\}$ if and only if there is $q \in S_{n-1}$ such that $\left(g_{i j}(\mathbf{a}), q\left(g_{s t}(\mathbf{a})\right)\right) \in E$.

Proof. We start with the "only if" part. Since $\sigma \in G(\mathbf{a}, p(\mathbf{a}))$, we have that $(\mathbf{a}, \sigma(\mathbf{a})) \in E$, and consequently,

$$
\left(g_{i j}(\mathbf{a}), g_{i j}(\sigma(\mathbf{a}))\right)=\left(g_{i j}(\mathbf{a}), p_{1}\left(g_{\sigma(i) \sigma(j)}(\mathbf{a})\right)\right) \in E
$$

for some $p_{1} \in S_{n-1}$, as required.

For the "if" part, assume to the contrary that for all $\sigma \in G(\mathbf{a}, p(\mathbf{a}))$ we have $\{\sigma(i), \sigma(j)\} \neq\{s, t\}$. We define a certain theory, $E_{1} \supseteq E$. We make use of the block $B$ of the element a in the remainder $\pi$ of $E$. Recall (Lemma 3.1) that up to equivalence $B$ is the unique minimal block of $\pi$, and $B=G_{B}\{\mathbf{a}\}$. It follows that all the sequences in the blocks of the orbit $[B]$ of $B$ are permutations of $\mathbf{a}$.

We shall write $\sum \mathbf{a}$ for $\sum_{i=1}^{n} a_{i}$. Let $J_{1}$ be the filter generated by all sequences $\mathbf{b}=\left(b_{1}, \ldots, b_{k}\right)$ such that either $\sum \mathbf{b}>\sum \mathbf{a}$ or else $\sum \mathbf{b}=\sum \mathbf{a}$ and $k<n-1$. Let $\pi_{1}$ be the equivalence relation on $\Gamma_{+} \backslash J_{1}$, whose nontrivial blocks are the sets in the orbit $[B]$ and two additional sets $B_{1}$ and $B_{2}$, defined as follows:

$$
B_{1}=\left\{f(\mathbf{b}): \mathbf{b}=g_{i j}(\sigma(\mathbf{a})), f \in S_{n-1}, \sigma \in G(\mathbf{a}, p(\mathbf{a}))\right\}
$$

and

$$
B_{2}=\left\{\mathbf{b}: \mathbf{b}=\left(b_{1}, \ldots, b_{n-1}\right), \sum \mathbf{b}=\sum \mathbf{a}, \mathbf{b} \notin B_{1}\right\} .
$$

Put $E_{1}=E\left(J_{1}, 0,1, \pi_{1}\right)$. To check that $E_{1}$ is a theory, one needs to check conditions $(\pi 1)-(\pi 4)$. The first three are trivial. For $(\pi 4)$ we need to check that if, for some $r, w, g_{r w}(B) \cap B_{1}$ is nonempty, then $g_{r w}(B) \subseteq B_{1}$. Without loss of generality it is enough to check that if $g_{r w}(\mathbf{a}) \in B_{1}$, then $g_{r w}(B) \subseteq B_{1}$. Using the fact that $\left\{a_{i}, a_{j}\right\} \neq\left\{a_{r}, a_{w}\right\}$ implies that $g_{i j}(\mathbf{a})$ is not equivalent to $g_{r w}(\mathbf{a})$, we get that 
$g_{r w}(\mathbf{a}) \in B_{1}$ if and only if there is $\sigma_{1} \in G(\mathbf{a}, p(\mathbf{a}))$ such that $\left\{\sigma_{1}(i), \sigma_{1}(j)\right\}=\{r, w\}$. But in this case $g_{r w}=p_{2} g_{i j} \sigma_{1}$ for some $p_{2} \in S_{n-1}$. Hence, if $g_{r w}(\mathbf{a}) \in B_{1}$, then $g_{r w}(B) \subseteq B_{1}$, as required.

Now, $E \subseteq E_{1}$, since $E$ is the minimal theory with the block $B$. Yet, $g_{s t}(\mathbf{a}) \in B_{2}$, and therefore there is no $q \in S_{n-1}$ such that $\left(g_{i j}(\mathbf{a}), q\left(g_{s t}(\mathbf{a})\right)\right) \in E_{1}$. Consequently, the same remains true for $E$.

We will say that a theory $E$ satisfies the condition $\mathcal{T}(\mathbf{a}, i, j, s, t)$ if $E$ is generated by a single permutational equation of the form $(\mathbf{a}, p(\mathbf{a}))$ for which there is $\sigma \in G(\mathbf{a}, p(\mathbf{a}))$ such that $\sigma(\{i, j\})=\{s, t\}$. Combining the lemma above with Corollary 3.2 we get the following.

Corollary 5.2. Let $\mathbf{a}=\left(a_{1}, \ldots, a_{n}\right)$, and let $i \neq j$ and $s \neq t$ be fixed positive integers not exceeding $n$. Then the set of join irreducible theories satisfying $\mathcal{T}(\mathbf{a}, i, j, s, t)$ is definable in $L(C o m)$.

Now we are prepared to prove the following

Theorem 5.3. Every join-irreducible theory generated by an equation of the form $(\mathbf{a}, p(\mathbf{a}))$ is individually definable.

The remainder of this section is devoted to the proof of this theorem. It consists of a number of steps, which are formulated in the form of lemmas below.

From the outset we assume that $\mathbf{a}=\left(a_{1}, \ldots, a_{n}\right), p \in S_{n}, E_{0}=E(\mathbf{a}, p(\mathbf{a}))$ is join-irreducible and $n \geq 2$. We define $\mathcal{T}$ to be the set of those join-irreducible theories $E=E(\mathbf{a}, q(\mathbf{a}))$ such that $E$ satisfies $\mathcal{T}(\mathbf{a}, i, j, s, t)$ for exactly the same tuples $i \neq j, s \neq t$ as $E_{0}=E(\mathbf{a}, p(\mathbf{a}))$ satisfies $\mathcal{T}(\mathbf{a}, i, j, s, t)$. By Corollary [5.2. $\mathcal{T}$ is definable. Our aim is to show that $\mathcal{T}$ consists of a single theory, and it is $E_{0}=E(\mathbf{a}, p(\mathbf{a}))$ that is the only member of $\mathcal{T}$. In each of the following lemmas we narrow the set of possible members of $\mathcal{T}$.

Our first claim concerns the structure of $G(\mathbf{a}, q(\mathbf{a}))$ (cf. Lemma 3.5). Let $\left\{Y_{1}\right.$, $\left.\ldots, Y_{k}\right\}$ be a partition of $X=\{1, \ldots, n\}$ such that $Y_{1}, \ldots, Y_{k}$ are the blocks of the least equivalence relation containing the orbit decomposition of $X$ under $G(\mathbf{a})$ and preserved by $p$.

Lemma 5.4. If $E(\mathbf{a}, q(\mathbf{a})) \in \mathcal{T}$, then the blocks of the least equivalence relation containing the orbit decomposition of $X$ under $G(\mathbf{a})$ and preserved by $q$ are exactly $Y_{1}, \ldots, Y_{k}$.

Proof. If all $a_{i}$ in a are different, then the least equivalence relation with demanded properties is the trivial relation, and in such a case there is nothing to prove. Hence, we may assume that there are $i \neq j$ with $\mathbf{a}_{i}=\mathbf{a}_{j}$.

First observe that if $q \in S_{n}$ and $Y_{1}^{\prime}, \ldots, Y_{k}^{\prime}$ are the blocks of the least equivalence relation containing the orbit decomposition of $X$ under $G(\mathbf{a})$ and preserved by $q$, then for any $s \neq t$ we have that $s$ and $t$ belong to the same block if and only if there are $i \neq j$ with $\mathbf{a}_{i}=\mathbf{a}_{j}$ and $\sigma \in G(\mathbf{a}, q(\mathbf{a}))$ with $\{\sigma(i), \sigma(j)\}=\{s, t\}$. To prove the lemma it is enough to show that whenever theories $E\left(\mathbf{a}, q_{1}(\mathbf{a})\right)$ and $E\left(\mathbf{a}, q_{2}(\mathbf{a})\right)$ induce different minimal equivalence relations $\sim_{1}, \sim_{2}$ containing the orbit decomposition of $X$ under $G(\mathbf{a})$ and preserved by $q_{1}$ and $q_{2}$, respectively, then there exist $s \neq t$ and $i \neq j$ such that exactly one of these theories satisfies $\mathcal{T}(\mathbf{a}, i, j, s, t)$.

To this end, let $s$ and $t$ be such that $s \sim_{1} t$ and $s \chi_{2} t$. By the note above, there exist $i \neq j$ such that $a_{i}=a_{j}$ and $\sigma \in G\left(\mathbf{a}, q_{1}(\mathbf{a})\right)$ with $\{\sigma(i), \sigma(j)\}=\{s, t\}$, and 
there is no $\tau \in G\left(\mathbf{a}, q_{2}(\mathbf{a})\right)$ with $\{\tau(i), \tau(j)\}=\{s, t\}$. Consequently, $E\left(\mathbf{a}, q_{1}(\mathbf{a})\right)$ satisfies $\mathcal{T}(\mathbf{a}, i, j, s, t)$, while $E\left(\mathbf{a}, q_{2}(\mathbf{a})\right)$ does not. This completes the proof of Lemma 5.4

The next claim concerns the induced action of the permutation $q$ on the sets $\left\{Y_{1}, \ldots, Y_{k}\right\}$ of blocks of the least equivalence relation containing the orbit decomposition of $X$ under $G(\mathbf{a})$ and preserved by $p$. Let $Z_{1}, \ldots, Z_{\ell}$ be the orbits of $G(\mathbf{a}, p(\mathbf{a}))$ in its action on $\left\{Y_{1}, \ldots, Y_{k}\right\}$.

Lemma 5.5. If $E(\mathbf{a}, q(\mathbf{a})) \in \mathcal{T}$, then the orbits of $G(\mathbf{a}, q(\mathbf{a}))$ in its action on $\left\{Y_{1}, \ldots, Y_{k}\right\}$ are exactly $Z_{1}, \ldots, Z_{\ell}$.

Proof. We can of course assume that if $n=2$, then $Y_{1}=\{1,2\}$. (Thus if $n=2$, then there is only the theory in $\mathcal{T}$ and there is nothing to prove.) Similarly as in the previous proof, we show that whenever groups $G\left(\mathbf{a}, q_{1}(\mathbf{a})\right)$ and $G\left(\mathbf{a}, q_{2}(\mathbf{a})\right)$ have different orbits in their actions on $\left\{Y_{1}, \ldots, Y_{k}\right\}$, then there are $i \neq j$ and $s \neq t$ such that exactly one of $E\left(\mathbf{a}, q_{1}(\mathbf{a})\right)$ or $E\left(\mathbf{a}, q_{2}(\mathbf{a})\right)$ satisfies $\mathcal{T}(\mathbf{a}, i, j, s, t)$. Suppose that for $u \neq v$ the sets $Y_{u}, Y_{v}$ belong to the same orbit of $G\left(\mathbf{a}, q_{1}(\mathbf{a})\right)$ and that they do not belong to the same orbit of $G\left(\mathbf{a}, q_{2}(\mathbf{a})\right)$. Choose $i \in Y_{u}$ and $s \in Y_{v}$. Then there are $j \in X \backslash\{i\}$ and $t \in X \backslash\{j\}$ such that $E\left(\mathbf{a}, q_{1}(\mathbf{a})\right)$ satisfies $\mathcal{T}(\mathbf{a}, i, j, s, t)$, and $E\left(\mathbf{a}, q_{2}(\mathbf{a})\right)$ does not. This completes the proof of Lemma 5.5.

The next restriction concerns the structure of permutation $q$. Let $p^{\prime}=c_{1} \cdots c_{\ell}$ be the cyclic decomposition of our fixed permutation $p$ in its action on the set $\left\{Y_{1}, \ldots, Y_{k}\right\}$, where $c_{i}$ is a cyclic permutation of $Z_{i}$.

Lemma 5.6. If $E(\mathbf{a}, q(\mathbf{a})) \in \mathcal{T}$, then $q$ acts on $Y_{1}, \ldots, Y_{k}$ as the product $q^{\prime}=$ $c_{1}^{t_{1}} \cdots c_{l}^{t_{\ell}}$ of such powers of the cycles $c_{1}, \ldots, c_{\ell}$ where $c_{i}$ and $c_{i}^{t_{i}}$ generate the same cyclic group for each $i$. (It is equivalent to saying that $q^{\prime}$ leaves every $Z_{i}$ invariant and the group generated by $q^{\prime}$, restricted to $Z_{i}$, is $\left\langle c_{i}\right\rangle$.)

Proof. By Lemma 5.5 since $E(\mathbf{a}, q(\mathbf{a})) \in \mathcal{T}, q^{\prime}$ acts on each $Z_{i}$ as some cycle (the trivial cycle if $\left|Z_{i}\right|=1$ ). If an orbit $Z_{i}$ has less than four elements, then there is a unique transitive cyclic group on $Z_{i}$; hence every $E \in \mathcal{T}$ has the required property with respect to such an orbit $Z_{i}$. Now consider an orbit $Z_{i}$ with more than three elements.

Choose $Y \in Z_{i}$. Then $q$ and $c_{i}$ induce the same cyclic group on $Z_{i}$ if and only if for every $j$ there are $s \in Y, t \in c_{i}(Y), u \in c_{i}^{j}(Y), w \in c_{i}^{j+1}(Y)$ such that $E(\mathbf{a}, q(\mathbf{a}))$ satisfies $\mathcal{T}(\mathbf{a}, s, t, u, w)$. Since this is true for $q=p$, by definition of $\mathcal{T}$, this is also true for every $q$ such that $E(\mathbf{a}, q(\mathbf{a})) \in \mathcal{T}$. The proof of Lemma 5.6 is thus completed.

Now, if all $Z_{i}$ are one-element sets, then the set $\mathcal{T}$ consists of exactly one theory, namely $E(\mathbf{a}, p(\mathbf{a}))$, and therefore $E(\mathbf{a}, p(\mathbf{a}))$ itself is individually definable. Hence, in the sequel, we can assume that one of $Z_{i}$ contains at least two elements. It follows that $p^{\prime}$ is nontrivial. Observe that, since $E=E(\mathbf{a}, p(\mathbf{a}))$ is join-irreducible, we may assume that the order of $p^{\prime}$ is a power of a prime. Indeed, otherwise $p^{\prime}=q_{1} q_{2}$ is a product of two permutations of relatively prime orders. Then $E$ is a join of two different theories generated by blocks $\left\langle G, q_{1}\right\rangle\{\mathbf{a}\}$ and $\left\langle G, q_{2}\right\rangle\{\mathbf{a}\}$, respectively, where $G=S_{Y_{1}} \times \cdots \times S_{Y_{k}}$. Consequently, the order of each $c_{i}$, which is equal to the cardinality $\left|Z_{i}\right|$, is $\delta^{m_{i}}$ for some $m_{i} \geq 0$ and a prime $\delta$. 
Our next restrictions concern the powers $t_{i}$ in a cyclic decomposition $q^{\prime}=$ $c_{1}^{t_{1}} \cdots c_{\ell}^{t_{\ell}}$ of the theories $E(\mathbf{a}, q(\mathbf{a}))$ which belong to $\mathcal{T}$. We may assume that, for all $i, \delta$ does not divide $t_{i}, 1 \leq t_{i}<\left|c_{i}\right|$, when $\left|c_{i}\right|>1$, and $t_{1}=1$ if $\left|c_{i}\right|=1$.

Lemma 5.7. Let $E(\mathbf{a}, q(\mathbf{a})) \in \mathcal{T}$ and suppose that $q$ acts on $Y_{1}, \ldots, Y_{k}$ as the product $q^{\prime}=c_{1}^{t_{1}} \cdots c_{\ell}^{t_{\ell}}$. If, for some $i, j,\left|c_{i}\right|=\left|c_{j}\right|$, then $t_{i}=t_{j}$.

Proof. Assume to the contrary that $t_{i} \neq t_{j}$. Let us denote $N=\left|c_{i}\right|=\delta^{m}$. By renumbering we can assume that $c_{i}=\left(Y_{1}, \ldots, Y_{N}\right)$ and $c_{j}=\left(Y_{N+1}, \ldots, Y_{2 N}\right)$. Fix $s_{1} \in Y_{1}, s_{2} \in Y_{2}, r_{1} \in Y_{N+1}$, and $r_{2} \in Y_{N+2}$. Then there is a permutation $\sigma \in G(\mathbf{a}, p(\mathbf{a}))$ such that $\sigma\left(s_{1}\right)=s_{2}, \sigma\left(r_{1}\right)=r_{2}$. Hence $\left\{\sigma\left(s_{1}\right), \sigma\left(r_{1}\right)\right\}=\left\{s_{2}, r_{2}\right\}$, and therefore $E(\mathbf{a}, p(\mathbf{a}))$ satisfies $\mathcal{T}\left(\mathbf{a}, s_{1}, r_{1}, s_{2}, r_{2}\right)$. Since $E(\mathbf{a}, q(\mathbf{a})) \in \mathcal{T}$, we have that $E(\mathbf{a}, q(\mathbf{a}))$ satisfies $\mathcal{T}\left(\mathbf{a}, s_{1}, r_{1}, s_{2}, r_{2}\right)$, i.e. there exists $\sigma_{1} \in\langle q, G(\mathbf{a})\rangle$ such that $\left\{\sigma_{1}\left(s_{1}\right), \sigma_{1}\left(r_{1}\right)\right\}=\left\{s_{2}, r_{2}\right\}$. The existence of $\sigma_{1} \in\langle q, G(\mathbf{a})\rangle$ with this property is possible only if there is $d>0$ such that $\left(c_{i}^{t_{i}} c_{j}^{t_{j}}\right)^{d}\left(Y_{1}\right)=Y_{2}$ and $\left(c_{i}^{t_{i}} c_{j}^{t_{j}}\right)^{d}\left(Y_{N+1}\right)=$ $Y_{N+2}$. Since $c_{i}$ acts regularly on the set $Z_{i}$, we have $d t_{i} \equiv 1(\bmod N)$. But then $d t_{j} \not \equiv 1(\bmod N)$, as $1 \leq t_{i}, t_{j}<N, t_{i} \neq t_{j}$ and $t_{i}$ and $t_{j}$ are prime to $N$. Finally, since $c_{j}$ acts regularly on the set $Z_{j}$, we have $c_{j}^{d t_{j}}\left(Y_{N+1}\right) \neq Y_{N+2}$. Hence there is no such $\sigma_{1}$, which leads to a contradiction and thus proves Lemma 5.7

We may without loss of generality assume that for every $i<n,\left|c_{i}\right| \leq\left|c_{i+1}\right|$. Note that where $E\left(\mathbf{a}, p_{1}(\mathbf{a})\right), E\left(\mathbf{a}, p_{2}(\mathbf{a})\right) \in \mathcal{T}$ we have $E\left(\mathbf{a}, p_{1}(\mathbf{a})\right)=E\left(\mathbf{a}, p_{2}(\mathbf{a})\right)$ if and only if $\left\langle p_{1}, G(\mathbf{a})\right\rangle=\left\langle p_{2}, G(\mathbf{a})\right\rangle$. Note also that we can choose $d$ so that $p_{1}^{d}$ acts as $c_{1}$ on $Z_{1}$. If $p_{2}=p_{1}^{d}$, then since $d$ is prime to $\delta$, we have that $\left\langle p_{2}, G(\mathbf{a})\right\rangle=\left\langle p_{1}, G(\mathbf{a})\right\rangle$. We choose $p_{2}$ from $\left\{\sigma:\langle\sigma, G(\mathbf{a})\rangle=\left\langle p_{2}, G(\mathbf{a})\right\rangle\right\}$ so that in the representation $p_{2}^{\prime}=$ $c_{1}^{t_{1}} \cdots c_{\ell}^{t_{\ell}}, t_{1}=t_{2}=\cdots=t_{j}=1$ for $j$ as large as possible. As a final step we prove

Lemma 5.8. If $E\left(\mathbf{a}, p_{2}(\mathbf{a})\right) \in \mathcal{T}$, then $p_{2}^{\prime}=p^{\prime}$.

Proof. Assume to the contrary that $p_{2}^{\prime} \neq p^{\prime}$. Let $i$ be minimal with $t_{i} \neq 1$. We know from the observation above that $i>1$. Assume that the permutations $c_{i-1}$ and $c_{i}$ act on the sets $\left\{Y_{1}, \ldots, Y_{N}\right\}$ and $\left\{Y_{s+1}, \ldots, Y_{s+M}\right\}$, respectively, where $N=\left|c_{i-1}\right|=\delta^{m_{i-1}}$, and $M=\left|c_{i}\right|=\delta^{m_{i}}$. We follow the proof of the previous lemma: $p\left(Y_{1}\right)=Y_{2}, p\left(Y_{s+1}\right)=Y_{s+2}$. Since $E\left(\mathbf{a}, p_{2}(\mathbf{a})\right) \in \mathcal{T}$, there is $d$ such that $p_{2}^{d}\left(Y_{1}\right)=Y_{2}$ and $p_{2}^{d}\left(Y_{s+1}\right)=Y_{s+2}$. This means that $c_{i-1}^{t_{i-1} d}\left(Y_{1}\right)=Y_{2}$ and $c_{i}^{t_{i} d}\left(Y_{s+1}\right)=Y_{s+2}$. Since $t_{i-1}=1$, we have, according to the first equality, that $d \equiv 1\left(\bmod \left|c_{i-1}\right|\right)$, where $\left|c_{i-1}\right|=\delta^{m_{i-1}}$. Since $\left|c_{j}\right|$ divides $\left|c_{j+1}\right|$ for all $j$, we have $d \equiv 1\left(\bmod \left|c_{j}\right|\right), j<i$. In particular $\delta$ does not divide $d$, hence $\left\langle p_{2}^{d}, G(\mathbf{a})\right\rangle=\left\langle p_{2}, G(\mathbf{a})\right\rangle$. Moreover, the second equality implies that $t_{i} d \equiv 1$ $\left(\bmod \left|c_{i}\right|\right)$, and consequently $t_{i} d \equiv 1\left(\bmod \left|c_{j}\right|\right)$ for all $j \leq i$. Thus we can replace $p_{2}$ by $p_{3}=p_{2}^{d}$ such that

$$
p_{3}^{\prime}=c_{1} \cdots c_{i} c_{i+1}^{s_{i+1}} \cdots c_{\ell}^{s_{\ell}}
$$

for some $s_{i+1}, \ldots, s_{\ell}$. This contradicts our maximality assumption for $p_{2}$.

Now, it follows from the last lemma that $\mathcal{T}$ is the one-element set consisting of $E(\mathbf{a}, p(\mathbf{a}))$, and since $\mathcal{T}$ is definable, it follows that $E(\mathbf{a}, p(\mathbf{a}))$ is individually definable. This completes the proof of Theorem 5.3. Using Corollary 4.2 we obtain

Corollary 5.9. If a theory $E$ is generated by a set of equations of the form $(\mathbf{a}, p(\mathbf{a}))$, then $E$ is definable in $L(C o m)$. 


\section{Regular equations With incomparable SEQuenCES}

Now we turn to the theories $E(\mathbf{a}, \mathbf{b})$ generated by a regular equation of the form $(\mathbf{a}, \mathbf{b})$, where $\mathbf{b}$ is incomparable with $\mathbf{a}$. In [9] it is shown that there are theories of this form which fail to be definable. We wish to find a complete characterization of these undefinable theories.

Let $B$ now denote the minimal block in the remainder of $E(\mathbf{a}, \mathbf{b})$ such that $\mathbf{a} \in B$. By Lemma 3.5. $G_{B}=G(\mathbf{a}, \mathbf{b})$ acts on the set $X=\{1, \ldots, n\}$ with orbits $Y_{1}, \ldots, Y_{k}$ as the product of the symmetric groups $S_{Y_{i}}$. We call $Y_{i}$ the orbits of the equation $(\mathbf{a}, \mathbf{b})$.

Lemma 6.1. Let $G_{B}=G(\mathbf{a}, \mathbf{b})=S_{Y_{1}} \times \cdots \times S_{Y_{k}}$ as above. The set of joinirreducible theories of the form $E(\mathbf{a}, p(\mathbf{b})), p \in S_{n}$, satisfying $G(\mathbf{a}, p(\mathbf{b}))=G_{B}$ is definable.

Proof. Observe that the set of theories mentioned in the lemma is exactly the set of those join-irreducible theories generated by an equation $(\mathbf{a}, p(\mathbf{b}))$ for some $p$ which contain the theory generated by the block $G_{B}\{\mathbf{a}\}$ and do not contain the theory generated by the block $H\{\mathbf{a}\}$ for any group $H$ strictly larger than $G_{B}$. The latter is definable by Corollary 5.9 .

Observe that $E(\mathbf{a}, \mathbf{b})=E(\mathbf{b}, \mathbf{a})$. Hence, in Lemma 6.1, we can exchange a with b. Now, it is not difficult to demonstrate that if the number of orbits $k>2$, then $E(\mathbf{a}, \mathbf{b})$ is definable.

Lemma 6.2. Let $E(\mathbf{a}, \mathbf{b})$ be a given join-irreducible theory with $G(\mathbf{a}, \mathbf{b})=S_{Y_{1}} \times$ $\ldots \times S_{Y_{k}}$ as above. If $k>2$, then $E(\mathbf{a}, \mathbf{b})$ is definable in $L(C o m)$.

Proof. By Lemma 6.1 and our remark following its proof, the set of those theories $E^{\prime}=E(\mathbf{a}, p(\mathbf{b}))$ with $G(\mathbf{a}, p(\mathbf{b}))=G_{B}$ and with $G\left(\mathbf{b}, p^{-1}(\mathbf{a})\right)=G_{B}$ is definable, and it includes $E=E(\mathbf{a}, \mathbf{b})$. Thus let $p \in S_{X}$ with $G(\mathbf{a}, p(\mathbf{b}))=G_{B}=$ $G\left(\mathbf{b}, p^{-1}(\mathbf{a})\right)$. Suppose that $E^{\prime}=E(\mathbf{a}, p(\mathbf{b}))$ and $E=E(\mathbf{a}, \mathbf{b})$ are different. We have to demonstrate that $E$ can be separated from $E^{\prime}$. It is easy to see that $G\left(\mathbf{b}, p^{-1}(\mathbf{a})\right)=G\left(p^{-1}(\mathbf{a}), \mathbf{b}\right)=p^{-1} G(\mathbf{a}, p(\mathbf{b})) p$. Thus we have that $p^{-1} G_{B} p=G_{B}$; equivalently, the permutation $p$ permutes the orbits $Y_{1}, \ldots Y_{k}$ of $(\mathbf{a}, \mathbf{b})$ (or respects the corresponding equivalence relation). The theory $E^{\prime}$ is generated by all the equations of the form $\left(\mathbf{a}, \sigma p_{1}(\mathbf{b})\right)$ where $\sigma$ ranges over $G_{B}$ and $p_{1}$ ranges over all permutations that permute the orbits and induce the same permutation on the orbits as does $p$.

Since $k>2$ and the permutation of the orbits induced by $p$ is not the identity, there are two cases.

Case 1. There are $i, j, k \in X,|\{i, j, k\}|=3$ such that $p\left(Y_{i}\right)=Y_{j}$ and $p\left(Y_{j}\right)=Y_{k}$. Choose $y_{i} \in Y_{i}, y_{j} \in Y_{j}, y_{k} \in Y_{k}$ with $p\left(y_{i}\right)=y_{j}, p\left(y_{j}\right)=y_{k}$. Let $\pi \in S_{X}$ be the permutation that exchanges $y_{i}$ and $y_{j}$ and fixes all other elements of $X$. Let $\lambda=p \pi p^{-1}$ so that $\lambda$ exchanges $y_{j}$ and $y_{k}$ and fixes all other elements. Now $E^{\prime} \vee E(\mathbf{a}, \lambda(\mathbf{a})) \geq E(\mathbf{b}, \pi(\mathbf{b}))$, since the equations

$$
\left(p^{-1}(\mathbf{a}), \mathbf{b}\right),(\lambda(\mathbf{a}), p \pi(\mathbf{b}))
$$

belong to $E^{\prime}$. But it is easy to show by induction on the length of equational deductions that if $q \in S_{X}$ and $(\mathbf{b}, q(\mathbf{a})) \in E \vee E(\mathbf{a}, \lambda(\mathbf{a}))$ or $(\mathbf{b}, q(\mathbf{b})) \in E \vee$ $E(\mathbf{a}, \lambda(\mathbf{a}))$, then $q\left(Y_{i}\right)=Y_{i}$. Thus $E(\mathbf{b}, \pi(\mathbf{b})) \not E E \vee E(\mathbf{a}, \lambda(\mathbf{a}))$. Thus we have a definable separation of $E$ from $E^{\prime}$. 
Case 2. There are $i, j, k \in X,|\{i, j, k\}|=3$ such that $p\left(Y_{i}\right)=Y_{j}$ and $p\left(Y_{j}\right)=Y_{i}$. Choose $z \in Y_{i}$, put $x=p(z)$, and choose $y \in Y_{k}$ and let $u=p^{-1}(y)$. Now define $\pi$ to be the permutation that exchanges $u$ and $z$ and fixes all other elements of $X$. Define $\lambda=p \pi p^{-1}$ so that $\lambda$ is the transposition on $x, y$. Now the same argument as in Case 1 works, showing that $E$ is separable from $E^{\prime}$.

Corollary 6.3. Let $E(\mathbf{a}, \mathbf{b})$ be an arbitrary theory such that $G(\mathbf{a}, \mathbf{b})$ has more than two orbits. Then $E(\mathbf{a}, \mathbf{b})$ is definable in $L(C o m)$.

Thus, only the case $k=2$ remains. We deal with a theory $E(\mathbf{a}, \mathbf{b})$ with precisely two orbits $Y_{1}, Y_{2}$. If $\left|Y_{1}\right| \neq\left|Y_{2}\right|$, then $E(\mathbf{a}, \mathbf{b})$ is the only join-irreducible theory of the form $E(\mathbf{a}, p(\mathbf{b}))$ with $G(\mathbf{a}, p(\mathbf{b}))=G\left(\mathbf{b}, p^{-1}(\mathbf{a})\right)=S_{Y_{1}} \times S_{Y_{2}}$, and Lemma 6.1 shows that $E(\mathbf{a}, \mathbf{b})$ is definable. Thus we can assume that $\left|Y_{1}\right|=\left|Y_{2}\right|$. Also, we get this corollary.

Corollary 6.4. Every theory $E(\mathbf{a}, \mathbf{b})$, where the common length of $\mathbf{a}$ and $\mathbf{b}$ is an odd integer, is definable in $L(C o m)$.

Hence, for the remainder of this section we may assume that the length of the incomparable sequences $\mathbf{a}$ and $\mathbf{b}$ is even, say, $2 n$, and without loss of generality we may assume that $Y_{1}=\{1, \ldots, n\}$ and $Y_{2}=\{n+1, \ldots, 2 n\}$. Referring in the sequel to the group $S_{n} \times S_{n}$ we always mean one where the factors act on $Y_{1}$ and $Y_{2}$, as above. In particular, we assume that $G(\mathbf{a}, \mathbf{b})=S_{n} \times S_{n}$. Without loss of generality, we can assume that $G(\mathbf{a}, \mathbf{b})$ preserves the sets $\{1, \ldots, n\}$ and $\{n+1, \ldots, 2 n\}$.

Lemma 6.5. With $\mathbf{a}, \mathbf{b}$ as above, if there exists a permutation $p \in S_{n} \times S_{n}$ such that $G(\mathbf{a}, p(\mathbf{b})) \neq S_{n} \times S_{n}$, then $E(\mathbf{a}, \mathbf{b})$ is definable.

Proof. Indeed, in such a case, since $G(\mathbf{a}, \mathbf{b})=S_{n} \times S_{n}$, then it follows from Corollary 6.3 and Corollary 5.9 , by an easy argument, that $E(\mathbf{a}, \mathbf{b})$ is the join of two definable theories $E(\mathbf{a}, p(\mathbf{b}))$ and $E(\mathbf{b}, p(\mathbf{b}))$.

Now define

$$
\tau_{n}=(1, n+1)(2, n+2) \cdots(n, 2 n) .
$$

With our previous work, we are reduced to discovering in which cases $E=E(\mathbf{a}, \mathbf{b})$ can be separated from a theory $E^{\prime}=E(\mathbf{a}, p(\mathbf{b}))$ where $G(\mathbf{a}, p(\mathbf{b}))=G\left(\mathbf{b}, p^{-1}(\mathbf{a})\right)=$ $S_{Y_{1}} \times S_{Y_{2}}$ and $E^{\prime} \neq E$. Here it is forced that $p$, like $\tau_{n}$, exchanges the sets $Y_{1}, Y_{2}$ and that $\tau_{n}=p q$ for some $q \in S_{Y_{1}} \times S_{Y_{2}}$. By Lemma 6.5. we can assume that where $\mathbf{b}^{\prime}=g(\mathbf{b}), G\left(\mathbf{a}, \mathbf{b}^{\prime}\right)=G(\mathbf{a}, \mathbf{b})$. This implies that $E\left(\mathbf{a}, \mathbf{b}^{\prime}\right)=E(\mathbf{a}, \mathbf{b})$ and $E\left(\mathbf{a}, \tau_{n}\left(\mathbf{b}^{\prime}\right)\right)=E(\mathbf{a}, p(\mathbf{b}))$. Thus, by changing notation (replacing the sequence $\mathbf{b}$ by $\left.\mathbf{b}^{\prime}\right)$, we are reduced to the situation where we have to discover whether $E(\mathbf{a}, \mathbf{b})$ can be separated from $E\left(\mathbf{a}, \tau_{n}(\mathbf{b})\right)$. Also, we always have the working assumption that $\mathbf{a}, \mathbf{b}$ are such that $G\left(\mathbf{a}, \tau_{n}(\mathbf{b})\right)=G\left(\mathbf{b}, \tau_{n}^{-1}(\mathbf{a})\right)=G(\mathbf{a}, \mathbf{b})=S_{n} \times S_{n}$.

Note that for a regular equation $(\mathbf{a}, \mathbf{b})$ with $\mathbf{a}, \mathbf{b}$ incomparable, either $E(\mathbf{a}, \mathbf{b})$ is a proper join of $E(\mathbf{a}, p(\mathbf{b}))$ and $E(\mathbf{b}, p(\mathbf{b}))$, for some $p$ (and then is definable) or $E(\mathbf{a}, \mathbf{b})$ is join-irreducible. By previous work, if $\mathbf{a}, \mathbf{b}$ are comparable or equivalent, then $E(\mathbf{a}, \mathbf{b})$ is definable. Thus, we have an interesting corollary, which however will not be used later.

Corollary 6.6. Every one-based theory, which is not join-irreducible, is definable. Consequently, under automorphisms of $L(C o m)$, one-based theories are mapped on one-based theories. 
In the sequel, all considered theories will be assumed to be join-irreducible. Lemma 6.5 proves very useful throughout the remainder of this section. Here is a second very useful lemma.

Lemma 6.7. Let $\mathbf{c}, \mathbf{d}, \mathbf{e}$ be incomparable members of $\Gamma_{+}$of length $2 n$ such that $G(\mathbf{c}) \cup G(\mathbf{d}) \cup G(\mathbf{e}) \subseteq S_{n} \times S_{n}$. If $E(\mathbf{c}, \mathbf{e})$ and $E(\mathbf{d}, \mathbf{e})$ are definable, then $E(\mathbf{c}, \mathbf{d})$ is definable, as well.

Proof. Let $E$ be the theory generated by the block

$$
\langle G(\mathbf{c}), G(\mathbf{d}), G(\mathbf{e})\rangle\{\mathbf{c}, \mathbf{d}, \mathbf{e}\} .
$$

Observe that $E=E(\mathbf{c}, \mathbf{e}) \vee E(\mathbf{d}, \mathbf{e})$, and therefore is definable. Yet, $E(\mathbf{c}, \mathbf{d}) \subseteq E$. Moreover, since $(\mathbf{c}, \mathbf{d}) \in E$ and $\langle G(\mathbf{c}), G(\mathbf{d}), G(\mathbf{e})\rangle \leq S_{n} \times S_{n}$, we have $(\mathbf{c}, p(\mathbf{d})) \notin E$ whenever $p \notin S_{n} \times S_{n}$. Now $E(\mathbf{c}, \mathbf{d})$ is the join of a finite number of join-irreducible theories of the form $E(\mathbf{c}, p(\mathbf{d})), p \in S_{2 n}$. For each of these theories $E\left(\mathbf{c}^{\prime}, \mathbf{d}^{\prime}\right)$ it is true that $G\left(\mathbf{c}^{\prime}, \mathbf{d}^{\prime}\right) \subseteq S_{n} \times S_{n}, E\left(\mathbf{c}^{\prime}, \mathbf{d}^{\prime}\right) \leq E$, and for any $q \notin S_{n} \times S_{n}, E\left(\mathbf{c}^{\prime}, q\left(\mathbf{d}^{\prime}\right)\right) \not E E$. Thus if $G\left(\mathbf{c}^{\prime}, \mathbf{d}^{\prime}\right) \neq S_{n} \times S_{n}$, then the equation has at least three orbits and it is definable, by Corollary 6.3 while if $G\left(\mathbf{c}^{\prime}, \mathbf{d}^{\prime}\right)=S_{n} \times S_{n}$, then $E\left(\mathbf{c}^{\prime}, \mathbf{d}^{\prime}\right)$ is separable from all the theories $E\left(\mathbf{c}^{\prime}, q\left(\mathbf{d}^{\prime}\right)\right)$ with $q(\{1, \ldots, n\})=\{n+1, \ldots, 2 n\}$, and then $E\left(\mathbf{c}^{\prime}, \mathbf{d},\right)$ is definable by Lemma 6.1. Thus finally, $E(\mathbf{c}, \mathbf{d})$ is the join of a finite number of definable theories, so it is definable.

6.1. Sequences with equal sums. Now we deal with some special cases starting from the case when $\sum \mathbf{a}=\sum \mathbf{b}$. For $n=1$, we have simply $\mathbf{a}=\left(a_{1}, a_{2}\right), \mathbf{b}=$ $\left(b_{1}, b_{2}\right), a_{1}+a_{2}=b_{1}+b_{2}$, and $a_{1} \neq a_{2}$, and $b_{1} \neq b_{2}$. Then, $\left|a_{1}-b_{1}\right|=\left|a_{2}-b_{2}\right| \neq$ $\left|a_{1}-b_{2}\right|=\left|a_{2}-b_{1}\right|$, and according to Theorem 2.4 (c), it follows immediately that $E(\mathbf{a}, \mathbf{b})$ is separable from $E\left(\mathbf{a}, \tau_{1}(\mathbf{b})\right)$, and so $E(\mathbf{a}, \mathbf{b})$ is definable. Hence we may assume that $n>1$.

In the sequel we often refer to the sequences with many occurrences of the same term. To simplify notation we will write that a sequence $\mathbf{a}=\left(a_{1}, \ldots, a_{n}\right) \in[k, n, m]$ (or is of type $[k, n, m]$ ) if $\mathbf{a}=(a, \ldots, a, b, \ldots, b, c, \ldots, c)$ with $a$ occurring $k$ times, $b$ occurring $n$ times, and $c$ occurring $m$ times. Note that the type as above determines the length of the sequence (to be equal to $k+n+m$ ); the equalities $a=b=c$ are not excluded.

Lemma 6.8. Let $\mathbf{a}=(k, 2, \ldots, 2,1, \ldots, 1) \in[1, n-1, n]$, and let $\mathbf{b}=\left(b_{1}, \ldots, b_{2 n}\right)$ be a sequence not equivalent to $\mathbf{a}$ such that $\sum \mathbf{b}=\sum \mathbf{a}$. Then the theory $E(\mathbf{a}, \mathbf{b})$ is definable.

Proof. We make use of Theorem 2.6. By virtue of this theorem, we may assume that there exist $i, j, u, w$ such that $g_{i j}(\mathbf{a})=p\left(g_{u w}(\mathbf{b})\right)$ for some permutation $p$.

First assume that $i=1$. Then we may also assume that $k>3$ (since otherwise $\mathbf{b}$ has to be equivalent to $\mathbf{a}$ ) and that (up to some permutation $p_{1}$ ) $\mathbf{b}=$ $(x, y, 2, \ldots, 2,1, \ldots, 1)$, where $x>2$, and 1 occurs at least $n-1$ times. If $x \neq y$, then the claim follows by Lemma 6.5. If $x=y$, then 2 occurs $n-2$ times in $\mathbf{b}$ (since otherwise we get a contradiction with the assumption that $\left.G(\mathbf{a}, \mathbf{b})=S_{n} \times S_{n}\right)$. Without loss of generality we may assume that $p_{1}$ is the identity. Then $m(E(\mathbf{a}, \mathbf{b}))>1$, while $m\left(E\left(\mathbf{a}, \tau_{n}(\mathbf{b})\right)\right)=1$, which proves the claim by Theorem 2.4 (b).

Hence, we may assume that both $i, j \neq 1$. If one of them is no greater than $n$, say, $1<i \leq n$, then $\mathbf{b}$ is equivalent to $(x, y, z, 2, \ldots, 2,1, \ldots, 1)$ when 1 occurs $n-1$ or $n$ times. In the latter case, we again get different values for $m(E)$, as above. In the former, $E(\mathbf{a}, \mathbf{b})$ is definable by Lemma 6.5 . 
Finally, if both $i, j>n$, then $\mathbf{b}$ has to be equivalent to $(x, y, 1, \ldots, 1,2, \ldots, 2)$, where 2 occurs $n$ times and $x+y=k$, and by Lemma 6.5 we may assume that $x=y$. Let $\mathbf{c}=(x+2, x, 2, \ldots, 2,1, \ldots, 1)$ where 1 occurs $n$ times. Then $E(\mathbf{a}, \mathbf{c})$ is definable by Lemma 6.5, and $E(\mathbf{b}, \mathbf{c})$ is definable by the same lemma, provided $n>2$. For $n=2$ we simply have $\mathbf{b}=(x, x, 2,2)$. Then it is enough to take $\mathbf{c}=(x+1, x+1,1,1)$. The definability of $E(\mathbf{a}, \mathbf{c})$ and $E(\mathbf{b}, \mathbf{c})$ then follows easily by comparing the parameters $m(E)$ and $r(E)$ for suitable theories. Using Lemma 6.7 completes the proof.

To complete the case $\sum \mathbf{a}=\sum \mathbf{b}$, if the conditions of Lemma 6.8 are not satisfied, take $\mathbf{c}=(k, 2, \ldots, 2,1, \ldots, 1) \in[1, n-1, n]$ with $k=\sum \mathbf{a}-3 n+2$. Obviously, sequences $\mathbf{a}, \mathbf{b}, \mathbf{c}$ are incomparable. By Lemma 6.8, $E(\mathbf{a}, \mathbf{c})$ and $E(\mathbf{b}, \mathbf{c})$ are definable, and using Lemma 6.7 we obtain:

Corollary 6.9. If $\sum \mathbf{a}=\sum \mathbf{b}$, then the theory $E(\mathbf{a}, \mathbf{b})$ is definable.

6.2. Sequences of the length 2. In this subsection we complete the case $n=$ 1. Then $E(\mathbf{a}, \mathbf{b})$ is generated by an equation $(\mathbf{a}, \mathbf{b})$, where the sequences $\mathbf{a}=$ $\left(a_{1}, a_{2}\right), \mathbf{b}=\left(b_{1}, b_{2}\right), a_{1} \neq a_{2}, b_{1} \neq b_{2}$, are incomparable. Denote $\tau=\tau_{1}$. We have to check if we can distinguish between join-irreducible theories $E(\mathbf{a}, \mathbf{b})$ and $E(\mathbf{a}, \tau(\mathbf{b}))$.

Since the case when $\sum \mathbf{a}=\sum \mathbf{b}$ has already been considered, we may assume that $\sum \mathbf{a}>\sum \mathbf{b}$. We may also assume without loss of generality that $a_{1}<a_{2}$ and $b_{1}<b_{2}$. It follows that $a_{1}>1$ (otherwise, $1+a_{2}=\sum \mathbf{a}>\sum \mathbf{b}$; that is, $a_{2} \geq \sum \mathbf{b}$, which contradicts incomparability). We start with the case when $b_{1}>1$.

Lemma 6.10. Let $\mathbf{a}, \mathbf{b}$ be as above. If $b_{1}>1$, then $E(\mathbf{a}, \mathbf{b})$ is definable.

Proof. Let $E$ be the theory generated by equations $\left(\left(a_{1}+2, a_{2}\right),\left(a_{1}+1, a_{2}\right)\right)$, $\left(\left(a_{1}, a_{2}+2\right),\left(a_{1}, a_{2}+1\right)\right)$, and $\left(\left(a_{1}, a_{2}, 1,1\right),\left(a_{1}, a_{2}, 1,2\right)\right)$. Since every equation has comparable sequences, $E$ is definable by Theorem 2.3 . Let us define $E_{1}=$ $E \vee E(\mathbf{a}, \mathbf{b})$, and $E_{2}=E \vee E(\mathbf{a}, \tau(\mathbf{b}))$. One can check easily that, since $a_{1}, a_{2}, b_{1}, b_{2}$ are all larger than 1 , the filter $J\left(E_{1}\right)=J\left(E_{2}\right)$ is generated by the following sequences: $\left(a_{1}+1, a_{2}\right),\left(b_{1}+1, b_{2}\right),\left(a_{1}, a_{2}+1\right),\left(b_{1}, b_{2}+1\right),\left(a_{1}, a_{2}, 1,1\right),\left(b_{1}, b_{2}, 1,1\right)$, and the one-element sequence $\left(a_{1}+a_{2}+1\right)$. Moreover, the only nontrivial blocks in $\pi\left(E_{1}\right)$ (up to equivalence) are $\left\{\left(a_{1}, a_{2}\right),\left(b_{1}, b_{2}\right)\right\}$ and $\left\{\left(a_{1}, a_{2}, 1\right),\left(b_{1}, b_{2}, 1\right)\right\}$, and in $\pi_{2}$ these are $\left\{\left(a_{1}, a_{2}\right),\left(b_{2}, b_{1}\right)\right\}$ and $\left\{\left(a_{1}, a_{2}, 1\right),\left(b_{2}, b_{1}, 1\right)\right\}$.

Let $E_{3}$ be the theory generated by equation $\left(\left(a_{1}, a_{2}, 1\right),\left(b_{1}, b_{2}, 1\right)\right)$. It is definable by Corollary 6.4. Now, it is easy to see that $E_{3} \subseteq E_{1}$, while $E_{3} \not \subset E_{2}$. This separates $E(\mathbf{a}, \mathbf{b})$ from $E(\mathbf{a}, \tau(\mathbf{b}))$, proving that both are definable.

Thus, it remains to consider the case $b_{1}=1$. Now, our equation is of the form $(\mathbf{a}, \mathbf{b})=\left(\left(a_{1}, a_{2}\right),\left(1, b_{2}\right)\right)$, and by incomparability, $a_{2}<b_{2}$; that is, $a_{2} \leq b_{2}-1$.

Assume first that $a_{2}<b_{2}-1$, and let $\mathbf{c}=\left(2, b_{2}-1\right)$. It is easy to see that $\mathbf{a}, \mathbf{b}, \mathbf{c}$ are incomparable. By Corollary 6.9, $E(\mathbf{b}, \mathbf{c})$ is definable, and by Lemma 6.10, $E(\mathbf{a}, \mathbf{c})$ is definable. Hence, by Lemma 6.7 $E(\mathbf{a}, \mathbf{b})$ is definable.

Thus we may assume that $a_{2}=b_{2}-1$. This means that the equation is of the form $\left(\left(a_{1}, a_{2}\right),\left(1, a_{2}+1\right)\right)$, where $2<a_{1}<a_{2}$ (the first inequality follows from $\Sigma \mathbf{a}>\Sigma \mathbf{b})$. If $a_{1}<a_{2}-2$, then we can take $\mathbf{c}=\left(a_{1}+1, a_{2}-1\right)$, and using a similar argument as above we show that $E(\mathbf{a}, \mathbf{b})$ is definable. (In the argument we need to apply the previous case rather than Lemma 6.10.) There remain the cases $a_{1}=a_{2}-2$ and $a_{1}=a_{2}-1$. 
If $a_{1}=a_{2}-1$ is even, then $\left(a_{2}+1\right)-a_{1}$ and $a_{2}-1$ are even while $a_{1}-1$ is odd. Then, by Theorem 2.4(c), $E(\mathbf{a}, \mathbf{b})$ is definable. The case when $a_{1}=a_{2}-1$ is odd has been considered in Section 7 of [9], where it was shown that in this very case we cannot separate $E(\mathbf{a}, \mathbf{b})$ from $E(\mathbf{a}, \tau(\mathbf{b}))$, and the theories are not definable.

The situation is similar for $a_{1}=a_{2}-2$. In this case, if $a_{1}+1$ is divisible by 3 , then $\left(a_{2}+1\right)-a_{1}$ and $a_{2}-1$ are both divisible by 3 , while $a_{1}-1$ is not divisible by 3. We proceed to show that if $a_{1}+1$ is not divisible by 3 , then $E(\mathbf{a}, \mathbf{b})$ and $E(\mathbf{a}, \tau(\mathbf{b}))$ are not definable. Substituting $a_{1}+1=k$ we have $\mathbf{a}=(k-1, k+1)$, $\mathbf{b}=(1, k+2)$, and $k$ is not divisible by 3 .

Lemma 6.11. Let $k>3$ be a fixed integer. If $E=E(J, m, r, \pi)$ is a theory containing exactly one of the equations $x y^{k+2}=x^{k-1} y^{k+1}$ or $x y^{k+2}=x^{k+1} y^{k-1}$, then the following hold:

(i) $(k+3),(1, k-1, k+1),(1,1, k+2) \in J$,

(ii) if $(\mathbf{a}, \mathbf{b}) \in \pi, \mathbf{a} \neq \mathbf{b}$, and $\mathbf{b}=(1, k+2)$, then either $\mathbf{a}=(k-1, k+1)$ or $\mathbf{a}=(k+1, k-1)$,

(iii) if $(\mathbf{a}, \mathbf{b}) \in \pi, \mathbf{a} \neq \mathbf{b}$, and $f$ is one of the operations $g_{i j}$ or $h_{1}$, then $f(\mathbf{a})$ is different from $(1, k+2)$ and $(k+2,1)$.

Proof. The proof is almost the same as that of Theorem 7.1 in [9]. The only difference is the need to consider the sequence $(k, k)$, which is however very easy to handle. As in the sequel we will have two similar lemmas (Lemma 6.15 and Lemma 6.23), we sketch the proof in this case, and omit the proof for the next two lemmas.

By assumption exactly one of $e_{1}=((k-1, k+1),(1, k+2))$ or $e_{2}=((k+1, k-$ $1),(1, k+2))$ is in $E$. Hence one of $e_{i}$ is in $\pi$. By $(\pi 3),(k+1, k-1,1),(k+2,1,1) \in J$, and by $(\pi 4),(k+3) \in J$, which proves (i).

For (ii), assume that it is the first equation that is in $E$ (the second case is analogous), which means that $(k-1, k+1)$ is in the class of $\mathbf{b}$. Assume to the contrary that $\mathbf{a}=\left(a_{1}, a_{2}\right) \neq(k-1, k+1)$. If $\mathbf{a}$ is equal to $(k+2,1)$ or $(k+1, k-1)$, then the second equation is in $E$ as well, a contradiction. Hence, a is not equivalent to, and therefore incomparable with, both $(1, k+2)$ and $(k-1, k+1)$. It follows that $a_{1}, a_{2} \leq k+1$, and consequently, $a_{1}, a_{2} \geq k$. The only possibility is $(k, k)$, but in this case we have: $((1, k+2),(k, k)) \in E$ and $((k-1, k+1),(k, k)) \in E$. Then obviously $((k, k),(k+1, k-1)) \in E$. Consequently, the equation $((1, k+2),(k+1, k-1))$ would be in $E$. Then again the second equation would be in $E$.

For (iii), for $f=h_{1}$ the claim is obvious, since the length of a is greater than 1 . So put $f=g_{i j}$, for some $i, j$, and assume to the contrary that $f(\mathbf{a})=(1, k+2)$ (the second case is analogous). Then, by (ii), $f(\mathbf{b})$ is equal to $(k-1, k+1)$ or $(k+1, k-1)$. Indeed, if $g_{i j}(\mathbf{b})=(1, k+2)$, then $\mathbf{a}=(1, t, k+2-t)$ and $\mathbf{b}=(1, s, k+2-s)$ for some $s, t$. Without loss of generality $s<t$. Then, $e_{1}=((1+t, k+2-t),(1+s, k+2-s)) \in$ $E$. Since $e_{2}=((k-1, k+1),(k-1+s-t, k+1+s-t))$ is a consequence of $e_{1}$, then $e_{2} \in E$. Hence, either $((1, k+2),(k-1+s-t, k+1+s-t)) \in E$ or $((k+2,1),(k-1+s-t, k+1+s-t)) \in E$. By $(\pi 4)$, in both cases $(k+2,1) \in J$, and both equations $((k+2,1),(k-1, k+1))$ and $((k+2,1),(k+1, k-1))$ belong to $E$, which contradicts the assumption.

Again we can consider only the case of $f(\mathbf{b})=(k-1, k+1)$. It follows that a has to be equivalent to $(1, k+2-r, r)$ and $\mathbf{b}$ has to be equivalent to $(k+1, k-1-s, s)$ or $(k+1-t, k-1, t)$. 
Now observe that for all $i, j, g_{i j}(\mathbf{a}) \notin J$, since the result is equivalent to $(1, k+2)$ or is less than $(k-1, k+1)$. Observe that $g_{1,3}(\mathbf{b}), g_{1,2}(\mathbf{b}) \in J$ when $s \leq 2$ and $s=1$, respectively. Similarly for the second case. This contradicts $(\pi 4)$.

Now, let $\Phi_{k}(E)$ denote the set of equations obtained from $E$ by exchanging equations $((1, k+2),(k-1, k+1))$ with $((1, k+2),(k+1, k-1))$.

Theorem 6.12. For every $E$ and $k>3$ not divisible by 3 , the set $\Phi_{k}(E)$ is a theory. Moreover, the operation $\Phi$ is an automorphism of $L(C o m)$.

Proof. By Lemma 6.11 exactly the same as in Section 7 of [9]. We note only that the condition that $k$ is odd is now replaced by the condition that $k$ is not divisible by 3 .

Let us conclude this section with the following

Corollary 6.13. Every join-irreducible theory generated by an equation $e=$ $((a, b),(c, d))$ is definable except for the cases when

(1) $e=((1, k+2),(k, k+1))$ or $e=((1, k+2),(k+1, k))$, for $k>2$ even,

(2) $e=((1, k+2),(k-1, k+1))$ or $e=((1, k+2),(k+1, k-1))$, for $k>3$ not divisible by 3 .

6.3. The smallest sequences. We now consider the case $n>1$. It is easy to prove, much as we did in a previous section, that if all $a_{i}, b_{i}>1$, then $E(\mathbf{a}, \mathbf{b})$ is definable. However it is not very helpful, since we still have to consider all the cases below which in fact also cover the cases with $a_{i}, b_{i}>1$. In this subsection we consider one more special nontrivial case when $\mathbf{a}$ is the smallest possible sequence having a term equal to $k+2$, and the group $G(\mathbf{a}) \subseteq S_{n} \times S_{n}$. This sequence has the form $\mathbf{a}=(k+2,2, \ldots, 2,1, \ldots, 1) \in[1, n-1, n]$, where $k>0$.

Lemma 6.14. Let $\mathbf{a}$ be as above, and let $\mathbf{b}=\left(b_{1}, \ldots, b_{2 n}\right)$ be incomparable with $\mathbf{a}$. Assume also that $\sum \mathbf{a}<\sum \mathbf{b}$. Then the theory $E(\mathbf{a}, \mathbf{b})$ is definable except for the case when either $n>2$ and $k$ is odd or $n=2$ and $k>3$ is odd and

$$
\mathbf{b}=(k, \ldots, k, k+1, \ldots, k+1) \quad \text { or } \quad \mathbf{b}=(k+1, \ldots, k+1, k, \ldots, k),
$$

with $k$ and $k+1$ occurring $n$ times each.

Proof. Let us denote $E=E(\mathbf{a}, \mathbf{b})$ and $E_{1}=E\left(\mathbf{a}, \tau_{n}(\mathbf{b})\right)$. (Recall that we maintain the assumption that $G(\mathbf{a}, \mathbf{b})=S_{n} \times S_{n}$.) If $k$ is even and $\mathbf{b}=(k, \ldots, k, k+$ $1, \ldots, k+1)$, then observe that $r(E)$ is even, while $r\left(E_{1}\right)=1$. Hence both $E$ and $E_{1}$ are definable. If $n=2$ and $k=3$, then it is easy to see that $(7,1,1) \in J\left(E_{1}\right)$ and $(7,1,1) \notin J(E)$. By Theorem 2.4(a), $E$ and $E_{1}$ are definable. Thus, we may assume that $\mathbf{b}$ is not equivalent to $(k+1, \ldots, k+1, k, \ldots, k)$. Obviously $b_{i}<k+2$ for all $i$, which implies that there exists $b_{j} \leq k-1$. Consequently, $k>1$.

Assume first that $k=2$. Then only 1,2 or 3 can occur in $\mathbf{b}$, and one of these numbers has to occur $n$ times. If it is 1 that occurs $n$ times, then $m(E) \neq m\left(E_{1}\right)$, and both the theories are definable. If 2 occurs $n$ times, then $r(E) \neq r\left(E_{1}\right)$, and again both the theories are definable. Hence, we may assume that neither 1 nor 2 occurs $n$ times. Suppose that 2 occurs $t<n$ times. We consider two cases.

If $n-t$ is even, then let $\mathbf{c}=(2, \ldots, 2,1, \ldots, 1,3, \ldots, 3) \in[n,(n-t) / 2,(n+t) / 2]$. Then $\sum \mathbf{b}=\sum \mathbf{c}$; moreover $\mathbf{c}$ is incomparable with $\mathbf{a}$ and $\mathbf{b}$. By Corollary 6.9, the case with $n$ occurrences of 2 and Lemma 6.7, we get that $E$ is definable. 
If $n-t$ is odd, then if $t=1$ or $t=n-1$ we are done by Lemma 6.5. Assume that $1<t<n-2$, and let $\mathbf{c}=(2, \ldots, 2,1,3, \ldots, 3) \in[n, 1, n-1]$. Also, a, $\mathbf{c}$ are incomparable, since $\sum \mathbf{c}>\sum \mathbf{b}>\sum \mathbf{a}$, and $\mathbf{b}, \mathbf{c}$ are incomparable since $\sum \mathbf{c}>$ $\sum \mathbf{b}$, and $\mathbf{b}$ has more terms equal to 3. By Lemma 6.5, $E(\mathbf{a}, \mathbf{c})$ is definable. To prove that $E(\mathbf{b}, \mathbf{c})$ is definable take $\mathbf{d}=(n+t+2,2, \ldots, 2,1, \ldots, 1) \in[1, n-1, n]$. Observe that $\sum \mathbf{d}=\sum \mathbf{b}<\sum \mathbf{c}(t<n-2)$; hence $\mathbf{d}$ is incomparable with $\mathbf{b}$ and $\mathbf{c}$. By Lemma 6.5 $E(\mathbf{c}, \mathbf{d})$ is definable. By Corollary 6.9, $E(\mathbf{b}, \mathbf{d})$ is definable. Hence, using Lemma 6.7. we get first that $E(\mathbf{b}, \mathbf{c})$ is definable and next that $E(\mathbf{a}, \mathbf{b})$ is definable, as required.

In the rest of the proof we assume that $k>2$. Denote $x=\left(\sum \mathbf{b}\right) /(2 n)$. We have $1.5<x<k+0.5$. Let $t$ be the integer part of $x$. We consider some subcases according to the value of the difference $x-t$.

If $x-t=0.5$, then $1<t<k$. Take

$$
\mathbf{c}=(t-1, t, \ldots, t, t+1, \ldots, t+1, t+2),
$$

where $t$ and $t+1$ occur $n-1$ times each. Since $t+2<k+2$ and $t+1, t+2>2$, we have that $\mathbf{c}$ is incomparable with $\mathbf{a}$. Since $\sum \mathbf{b}=\sum \mathbf{c}$, the sequences $\mathbf{b}, \mathbf{c}$ are incomparable or equivalent. In the latter case we are done by Lemma 6.5. In the former, we apply Lemma 6.7, observing that $E(\mathbf{a}, \mathbf{c})$ is definable by Lemma 6.5 and $E(\mathbf{b}, \mathbf{c})$ is definable by Corollary 6.9

If $x-t>0.5$, then we apply the same method, but we choose different sequences c depending on the value of $t$. If $t=k-1$, then we take

$$
\mathbf{c}=(k-2, k-1, \ldots, k-1, k, \ldots, k, k+1, \ldots, k+1) \in[1, n-1, n-m, m],
$$

where $m=\sum \mathbf{b}-(2 k-1) n+1$. If $t<k-1$, then we take

$$
\mathbf{c}=(t+2, t+1, \ldots, t+1, t, \ldots, t) \in[1, n-1, n],
$$

provided that $\sum \mathbf{b}=(2 t+1) n+1$. If $\sum \mathbf{b}>(2 t+1) n+1$, then we take

$$
\mathbf{c}=(t+3, t+2, \ldots, t+2, t+1, \ldots, t+1, t, \ldots, t) \in[1, m, n-m-1, n],
$$

where $m=\sum \mathbf{b}-(2 t+1) n-2$. We leave it to the reader to check that in each of these cases the argument as above works, as well. In the final case the argument $x-t<0.5$ is symmetric to that above and is also left to the reader.

The exceptional case of Lemma 6.14 leads to new automorphisms of $L(\mathrm{Com})$. First we have

Lemma 6.15. Let $k>2$ be odd, $n>1$, and $k>3$ if $n=2$. Let $\mathbf{a}=(k+$ $2,2, \ldots, 2,1, \ldots, 1) \in[1, n-1, n]$ and $\mathbf{b}=(k, \ldots, k, k+1, \ldots, k+1) \in[n, n]$, and let $B_{1}=\left(S_{n} \times S_{n}\right)\{\mathbf{a}, \mathbf{b}\}$ and $B_{2}=\left(S_{n} \times S_{n}\right)\left\{\mathbf{a}, \tau_{n}(\mathbf{b})\right\}$. If $E=E(J, m, r, \pi)$ is a theory containing exactly one of the blocks $B_{1}$ or $B_{2}$, then the following hold:

(i) All the sequences

$$
\begin{aligned}
& (k+3,2, \ldots, 2,1, \ldots, 1) \in[1, n-1, n-1], \\
& (k+4,2, \ldots, 2,1, \ldots, 1) \in[1, n-2, n], \\
& (k+2,2, \ldots, 2,1, \ldots, 1) \in[1, n, n-2], \\
& (k+2,2, \ldots, 2,1, \ldots, 1) \in[1, n-1, n+1], \\
& (1, k, \ldots, k, k+1, \ldots, k+1) \in[1, n, n], \text { and } \\
& (k+2,4,2, \ldots, 2,1, \ldots, 1] \in[1,1, n-3, n], \text { if } n>2,
\end{aligned}
$$
belong to $J$.

(ii) There is $i=1$ or 2 such that for every $\mathbf{c}$, if $(\mathbf{a}, \mathbf{c}) \in \pi$, then $\mathbf{c} \in B_{i}$. 
(iii) If $f$ is one of the operations $g_{i j}$ or $h_{1}$ and $B$ is a block in $\pi$, then $f(B) \subseteq B_{1}$ implies that $f(B) \subseteq B_{2}$ or $f(B) \subseteq \tau_{n}\left(B_{2}\right)$, and similarly $f(B) \subseteq B_{2}$ implies that $f(B) \subseteq B_{1}$ or $f(B) \subseteq \tau_{n}\left(B_{1}\right)$.

The proof of this lemma is analogous to that of Lemma 6.11. Also, as in Theorem 6.12 (following again the argument in Section 7 of [9]) we get the next result on automorphisms. Note that if a theory $E$ contains equation $(\mathbf{a}, \mathbf{b})$, then it contains all the equations from the block $B_{1}$, and if it contains equation $\left(\mathbf{a}, \tau_{n}(\mathbf{b})\right)$, then it contains all the equations from the block $B_{2}$. By $\Phi_{k}(E)$ we denote the set of equations obtained from $E$ by exchanging all the equations corresponding to the block $B_{1}$ with all the equations corresponding to the block $B_{2}$. Then we have

Theorem 6.16. For $k>2$ odd and every theory $E$, if either $n>2$ or $n=2$ and $k>3$, then the set $\Phi_{k}(E)$ is a theory. The mapping $\Phi_{k}$ is an automorphism of $L(\mathrm{Com})$.

Now we consider the case where $\sum \mathbf{a}>\sum \mathbf{b}$.

Lemma 6.17. Let $\mathbf{a}=(k+2,2, \ldots, 2,1, \ldots, 1) \in[1, n-1, n], \mathbf{b}=\left(b_{1}, \ldots, b_{2 n}\right)$ be incomparable with $\mathbf{a}$, and $\sum \mathbf{a}>\sum \mathbf{b}$. Then $E(\mathbf{a}, \mathbf{b})$ is definable.

Proof. Assume that $b_{1}$ is the largest term in $\mathbf{b}$. If $b_{1}>b_{i}$ for all $i \neq 1$, then by Lemma $6.5 E(\mathbf{a}, \mathbf{b})$ is definable. Hence we can assume $b_{2}=b_{1}$.

Assume first that $b_{1}>4$. Then in the case where there is no $i>n$ with $b_{i}=b_{1}-1$ we take $\mathbf{c}=\left(b_{1}+1, b_{2}-1, b_{3}, \ldots, b_{2 n}\right)$. If there exists such an $i$, but there is no $j>n$ with $b_{j}=b_{1}-2$, we take $\mathbf{c}=\left(b_{1}+2, b_{2}-2, b_{3}, \ldots, b_{2 n}\right)$. Otherwise, we take $\mathbf{c}=\left(b_{1}+1, b_{2}, \ldots, b_{i-1}, b_{i}-1, b_{i+1}, \ldots, b_{2 n}\right)$, where $b_{i}=b_{1}-1$. In each case $\sum \mathbf{c}=\sum \mathbf{b}$, hence $\mathbf{b}, \mathbf{c}$ are incomparable. Moreover, the sum of all terms in $\mathbf{b}$ greater than 2 equals the sum of all terms in $\mathbf{c}$ greater than 2 since $b_{1}=b_{2}>4$, and the two sequences agree on remaining terms. From this it easily follows that $\mathbf{c} \not \mathbf{a}$ since $\mathbf{b} \not \leq \mathbf{a}$ and each of $\mathbf{b}, \mathbf{c}$ have fewer than $n$ occurrences of 2 . Hence $\mathbf{c}$ and a are incomparable. By Lemma 6.5, the theory $E(\mathbf{a}, \mathbf{c})$ is definable. By Corollary 6.9, the theory $E(\mathbf{b}, \mathbf{c})$ is definable. Hence by Lemma 6.7, we obtain that $E(\mathbf{a}, \mathbf{b})$ is definable.

The cases when $b_{1}=4$ or $b_{1}=3$ are a little bit harder, and we consider them separately. First, we assume that $b_{1}=4$, and we consider various possibilities with regard to how many times the numbers $4,3,2,1$ occur in $\mathbf{b}$. Let $t_{i}$ denote the number of occurrences of $i$ in $\mathbf{b}$, for $i \leq 4$.

If $t_{1}=n$ or $t_{2}=n$, then we take $\mathbf{c}=\left(5,3, b_{3}, \ldots, b_{2 n}\right)$ and follow the proof above. Now suppose that $t_{1}+t_{2}=n$ and $t_{1}>0, t_{2}>0$. Of course, we assume that $t_{1}>1$ and $t_{2}>1$ (otherwise Lemma 6.5 applies). There are three cases to consider. In the first case, we have $\mathbf{b}=(4, \ldots, 4,3, \ldots, 3,1, \ldots, 1,2, \ldots, 2) \in\left[t_{4}, t_{3}, t_{1}, t_{2}\right]$ where $t_{4}+t_{3}=n, t_{4} \geq 2, t_{1} \geq 2, t_{2} \geq 2$ and $t_{3} \geq 2$ if $t_{3} \neq 0$. Since $\mathbf{b} \not \leq \mathbf{a}$ and $t_{2}<n$, then $4 t_{4}+3 t_{3}>k+2$. Put $\mathbf{c}=(5,4, \ldots, 4,3, \ldots, 3,2, \ldots, 2,1, \ldots, 1) \in$ $\left[1, t_{4}-2, t_{3}+1, t_{1}, t_{2}\right]$. Now $\sum \mathbf{c}=\sum \mathbf{b}$ and the sum of terms that exceed 2 in $\mathbf{c}$ is greater than $k+2$, so $\mathbf{a}$ and $\mathbf{c}$ are incomparable as well as $\mathbf{b}$ and $\mathbf{c}$. We have that $E(\mathbf{b}, \mathbf{c})$ is definable by Corollary 6.9 and $E(\mathbf{a}, \mathbf{c})$ is definable by Lemma 6.5, so that $E(\mathbf{a}, \mathbf{b})$ is definable by Lemma 6.7

Still assuming that $t_{1}+t_{2}=n$ and $t_{1}>0, t_{2}>0$, the next two cases are where the entries equal to 1 in $\mathbf{b}$ occur in the opposite orbit of the equation than the entries equal to 2 . In the second case we have that (up to permutation in 
$G(\mathbf{a}, \mathbf{b})) \mathbf{b}=(4, \ldots, 4,2, \ldots, 2,1, \ldots, 1,3, \ldots, 3) \in\left[t_{1}, t_{2}, t_{1}, t_{2}\right]$. In this case, we have that $4 t_{1}+3 t_{2}>k+2$, and we put $\mathbf{c}=(9,5,4, \ldots, 4,2, \ldots, 2,1, \ldots, 1,3, \ldots, 3) \in$ $\left[1,1, t_{1}-2, t_{2}, t_{1}+2, t_{2}-2\right]$. Here we have that $\sum \mathbf{c}=\sum \mathbf{b}+2$, and with some effort it can be seen that $\mathbf{b} \not \mathbf{c}$, so that $\mathbf{b}$ and $\mathbf{c}$ are incomparable. Moreover, note that $G(\mathbf{b}, \mathbf{c})$ has three orbits, so that $E(\mathbf{b}, \mathbf{c})$ is definable by Lemma 6.5. The sum of terms exceeding 2 in $\mathbf{c}$ is the same as in $\mathbf{b}$, and exceeds $k+2$, so $\mathbf{c} \not \leq \mathbf{a}$. Also, we have that $\mathbf{a} \not \leq \mathbf{c}$, since $\sum \mathbf{a}>\sum \mathbf{b}$ implies that $k+2>2 n+2 \geq 10$. The theory $E(\mathbf{a}, \mathbf{c})$ is then definable by Lemma 6.5. Thus $E(\mathbf{a}, \mathbf{b})$ is definable by Lemma 6.7

Still assuming that $t_{1}+t_{2}=n$ and $t_{1}>0, t_{2}>0$, the third case is where $\mathbf{b}=$ $(4, \ldots, 4,1, \ldots, 1,2, \ldots, 2,3, \ldots, 3) \in\left[t_{2}, t_{1}, t_{2}, t_{1}\right]$. Here we take $\mathbf{c}=(9,5,4, \ldots$, $4,1, \ldots, 1,2, \ldots, 2,3, \ldots, 3) \in\left[1,1, t_{2}-2, t_{1}, t_{2}+2, t_{1}-2\right]$. Note that in this case $\sum \mathbf{a}>\sum \mathbf{b}$ is equivalent to $k>n+2 t_{2} \geq 8$, so that $k+2 \geq 10$. The proof now follows the lines in the last paragraph, noting again that $G(\mathbf{b}, \mathbf{c})$ has three orbits.

Thus the proof for the case that $b_{\max }=b_{1}=4$ and $t_{1}+t_{2}=n$ is finished.

Now we consider possibilities for $t_{3}$. If $t_{3}=n$ and $t_{2}>0$, we take $\mathbf{c}=$ $(5,4, \ldots, 4,2, \ldots, 2,1, \ldots, 1,3, \ldots, 3) \in\left[1, t_{4}-1, t_{2}-1, t_{1}+1, n\right]$. If $t_{3}<n$ and $t_{1}+t_{2}<n$, we take $\mathbf{c}=\left(4+n-t_{3}, 4, \ldots, 4,1, \ldots, 1,2, \ldots, 2,3, \ldots, 3\right) \in$ $\left[1, t_{4}+t_{3}-n-1, t_{1}, t_{2}, n\right]$. If $t_{3}<n$ and $t_{1}+t_{2}>n$, then take $\mathbf{c}=(4+n-$ $\left.t_{1}, 4, \ldots, 4,3, \ldots, 3,2, \ldots, 2,1, \ldots, 1\right) \in\left[1, t_{4}-1, t_{3}, t_{2}+t_{1}-n, n\right]$. In every case we follow the same proof as above.

Thus for $b_{\max }=b_{1}=4$ it remains to be the case where $t_{3}=n$ and $t_{2}=0$. Here, we need to distinguish two subcases: $t_{1}=0$ or $t_{1}>0$. If $t_{1}=0$, then

$$
\mathbf{b}=(4, \ldots, 4,3, \ldots, 3) \in[n, n] .
$$

If $k+2<7 n-1$, then we take

$$
\mathbf{c}=(7,4, \ldots, 4,1,3, \ldots, 3) \in[1, n-2,1, n] .
$$

We have that $\sum \mathbf{c}=\sum \mathbf{b}$ and $\mathbf{b}, \mathbf{c}$ are incomparable, and therefore $E(\mathbf{b}, \mathbf{c})$ is definable. Since $\sum \mathbf{a}>\sum \mathbf{c}$ and $7+4(n-1)+3 n=7 n-1>k+2$, a, $\mathbf{c}$ are incomparable. Hence, by Lemma 6.5, $E(\mathbf{a}, \mathbf{b})$ is definable.

If $k+2=7 n-1$, we take

$$
\mathbf{d}=(2, \ldots, 2, k+1,1, \ldots, 1) \in[n, 1, n-1] .
$$

Observe that $\sum \mathbf{d}=\sum \mathbf{a}$, and therefore $\mathbf{a}, \mathbf{d}$ are incomparable, and $E(\mathbf{a}, \mathbf{d})$ is definable. In turn, $\mathbf{b}, \mathbf{d}$ are incomparable, since $\sum \mathbf{d}>\sum \mathbf{b}$, and $7 n>7 n-2=k+1$. Take $\mathbf{c}$ exactly as above. We have that $\sum \mathbf{c}=\sum \mathbf{b}$ and $\mathbf{b}, \mathbf{c}$ are incomparable, so $E(\mathbf{b}, \mathbf{c})$ is definable. Moreover, $\sum \mathbf{d}>\sum \mathbf{c}$ and $7+4(n-2)+3 n=7 n-1>k+1$, and therefore $\mathbf{d}, \mathbf{c}$ are incomparable. By Lemma 6.5 $E(\mathbf{d}, \mathbf{c})$ is definable. Hence, using Lemma 6.7. we get first that $E(\mathbf{b}, \mathbf{d})$ is definable and next that $E(\mathbf{a}, \mathbf{b})$ is definable, as required.

It remains the case where $b_{\max }=b_{1}=4, t_{3}=n, t_{2}=0$, and $t_{1}>0$. Here,

$$
\mathbf{b}=(4,4, \ldots, 4,1, \ldots, 1,3, \ldots, 3) \in\left[t_{4}, t_{1}, n\right] .
$$

Since $t_{2}=0$ and $\mathbf{b} \not \leq \mathbf{a}$, then $4 t_{4}+3 t_{3}>k+2$. As usual, we can assume that $t_{4}>1$ and $t_{1}>1$. Let $\mathbf{e}=(6,2,4, \ldots, 4,1, \ldots, 1,3, \ldots, 3) \in\left[1,1, t_{4}-2, t_{1}, n\right]$. Here $\sum \mathbf{e}=\sum \mathbf{b}$ and $\mathbf{e}, \mathbf{b}$ are incomparable, so $E(\mathbf{b}, \mathbf{e})$ is definable. If $\mathbf{e}$ and $\mathbf{a}$ are incomparable, then $E(\mathbf{a}, \mathbf{e})$ is definable by Lemma 6.5 and then $E(\mathbf{a}, \mathbf{b})$ is definable by Lemma 6.7. So we assume that $\mathbf{e}<\mathbf{a}$. This implies that $4 t_{4}+3 n-2$ (the sum 
of terms exceeding 2 in $\mathbf{e}$ ) is no greater than $k+2$. Since also $k+2<4 t_{4}+3 n$, we have that

$$
k+2=4 t_{4}+3 n-2 \text { or } k+2=4 t_{4}+3 n-1 .
$$

Put

$$
\mathbf{c}=\left(3, \ldots, 3,1, \ldots, 1, t_{4}+3 n-5,5,2, \ldots, 2\right) \in\left[t_{4}, t_{1}, 1,1, n-2\right] .
$$

Note that $t_{4}+3 n-5=4 t_{4}+3 t_{1}-5 \geq 9$, since $t_{4} \geq 2$ and $t_{1} \geq 2$. We have $\mathbf{c} \not \leq \mathbf{b}$, obviously, and it can be shown that $\mathbf{b} \not \mathbf{c}$. The group $\mathrm{G}(\mathbf{b}, \mathbf{c})$ has three orbits, so $E(\mathbf{b}, \mathbf{c})$ is definable (by Lemma 6.5). The sum of terms greater than 2 in $\mathbf{c}$ is the same as in $\mathbf{b}$, so $\mathbf{c} \not \mathbf{a}$. We have $\mathbf{a} \not \leq \mathbf{c}$ since $k+2>t_{4}+3 n-5>5$. Thus the theory $E(\mathbf{a}, \mathbf{c})$ is definable by Lemma 6.5 and $E(\mathbf{a}, \mathbf{b})$ is definable by Lemma 6.7 This completes the proof for the case $b_{\max }=4$.

Finally, we consider the case $b_{\max }=3$. As usual we may assume $t_{3}>1$. One of the three numbers $t_{1}, t_{2}, t_{3}$ must be $n$, so we have three cases to consider:

$$
\begin{gathered}
\mathbf{b}=\mathbf{b}_{1}=(3, \ldots, 3,2, \ldots, 2,1, \ldots, 1) \in\left[t_{3}, t_{2}, n\right], \\
\text { or } \mathbf{b}=\mathbf{b}_{2}=(3, \ldots, 3,1, \ldots, 1,2, \ldots, 2) \in\left[t_{3}, t_{1}, n\right], \\
\text { or } \mathbf{b}=\mathbf{b}_{3}=(3, \ldots, 3,1, \ldots, 1,2, \ldots, 2) \in\left[n, t_{1}, t_{2}\right] .
\end{gathered}
$$

Since $m\left(E\left(\mathbf{a}, \mathbf{b}_{1}\right)\right)=2$ while $m\left(E\left(\mathbf{a}, \tau_{n}\left(\mathbf{b}_{1}\right)\right)\right)=1$, it follows that $E\left(\mathbf{a}, \mathbf{b}_{1}\right)$ is separable from $E\left(\mathbf{a}, \tau_{n}\left(\mathbf{b}_{1}\right)\right)$ and so $E\left(\mathbf{a}, \mathbf{b}_{1}\right)$ is definable.

Put $\mathbf{c}_{2}=(4, \ldots, 4,2, \ldots, 2,1, \ldots, 1) \in\left[t_{3}, t_{1}, n\right]$. Now $\sum \mathbf{c}_{2}=\sum \mathbf{b}_{2}$ and of course $\mathbf{c}_{2}, \mathbf{b}_{2}$ are incomparable, so $E\left(\mathbf{c}_{2}, \mathbf{b}_{2}\right)$ is definable. Since $\mathbf{b} \not \mathbf{a}$, we have $3 t_{3}+2>k+2$. Therefore, $4 t_{3} \geq 3 t_{3}+2>k+2$. Consequently, $\mathbf{c}_{2} \not \leq \mathbf{a}$. Thus $\mathbf{c}_{2}, \mathbf{a}$ are incomparable. Moreover, $m\left(E\left(\mathbf{a}, \mathbf{c}_{2}\right)\right)=2$ and $m\left(E\left(\mathbf{a}, \tau\left(\mathbf{c}_{2}\right)\right)\right)=1$. Thus $E\left(\mathbf{a}, \mathbf{c}_{2}\right)$ is definable. Finally, it follows that $E\left(\mathbf{a}, \mathbf{b}_{2}\right)$ is definable, by Lemma 6.7 .

If $t_{2}=n$ in $\mathbf{b}_{3}$, then $\mathbf{b}_{3}$ falls under the $\mathbf{b}_{2}$ case. If $t_{2}=0$ in $\mathbf{b}_{3}$, then $\mathbf{b}_{3}$ is in the $\mathbf{b}_{1}$ case. Thus assume that $0<t_{2}<n$. Let $\mathbf{c}_{3}=(4,3, \ldots, 3,1, \ldots, 1,2, \ldots, 2) \in$ $\left[1, n-1, t_{1}+1, t_{2}-1\right]$. Here $\sum \mathbf{c}_{3}=\sum \mathbf{b}_{3}$, and clearly $\mathbf{c}_{3}, \mathbf{b}_{3}$ are incomparable. Thus $E\left(\mathbf{c}_{3}, \mathbf{b}_{3}\right)$ is definable. The sum of the terms that exceed 2 in $\mathbf{c}_{3}$ is one greater than the sum in $\mathbf{b}_{3}$, and thus $\mathbf{c}_{3} \not \leq \mathbf{a}$ because there are fewer than $n$ terms equal to 2 (in $\mathbf{c}_{3}$ as in $\mathbf{b}_{3}$ ). It follows that $\mathbf{a}, \mathbf{c}_{3}$ are incomparable and $E\left(\mathbf{a}, \mathbf{c}_{3}\right)$ is definable by Lemma 6.7. Finally, $E\left(\mathbf{a}, \mathbf{b}_{3}\right)$ is definable by Lemma 6.7 This concludes our proof of Lemma 6.17

6.4. Other cases. Now we are ready to consider all the remaining cases. In view of the results established above we may assume that, say, $\sum \mathbf{a}>\sum \mathbf{b}, n>1$, and $\mathbf{a}$ and $\mathbf{b}$ are different from $(k+2,2, \ldots, 2,1, \ldots, 1) \in[1, n-1, n]$. Recall that we still work under the assumption that the symmetry groups of considered sequences are contained in $S_{n} \times S_{n}$. This means, in particular, that there are at most $n$ occurrences of the same term in the sequence.

Lemma 6.18. Let $\mathbf{a}$ and $\mathbf{b}$ be incomparable sequences of the length $2 n$ with $\sum \mathbf{a}>$ $\sum \mathbf{b}$. If 2 occurs exactly $n$ times in $\mathbf{b}$, then $E(\mathbf{a}, \mathbf{b})$ is definable.

Proof. We may assume that there is at least one $i$ such that $b_{i}>2$ (otherwise $\mathbf{b}<\mathbf{a})$.

First assume there is only one such $i$, so that we have

$$
\mathbf{b}=(b, 1, \ldots, 1,2, \ldots, 2) \in[1, n-1, n],
$$


with $b>2$. Let $\mathbf{c}=(b+1,2 \ldots, 2,1, \ldots, 1) \in[1, n-1, n]$. Now $\sum \mathbf{c}=\sum \mathbf{b}<\sum \mathbf{a}$. If all $a_{i} \leq b$, then $\mathbf{c}$ and $\mathbf{a}$ are incomparable. Then $E(\mathbf{a}, \mathbf{b})$ is definable by Lemma 6.7 and Lemma 6.14, since a equivalent to $(b-1, \ldots, b-1, b, \ldots, b) \in[n, n]$ would entail $\mathbf{b} \leq \mathbf{a}$. Certainly, all $a_{i}<b+2$, otherwise $\mathbf{b}<\mathbf{a}$. Thus we are reduced to the case where the largest $a_{i}$ is $b+1$. If $b+1$ occurs twice in $\mathbf{a}$, then again, $\mathbf{b}<\mathbf{a}$. Thus $a_{\max }=b+1$ occurs just once in $\mathbf{a}$. But now, the definability of $E(\mathbf{a}, \mathbf{b})$ follows from Lemma 6.5.

Thus we can now assume that $\mathbf{b}=\left(b_{1}, b_{2}, \ldots, 2, \ldots, 2\right)$ where $b_{1} \geq b_{2} \geq 3$. Let $k$ be the sum of all terms in $\mathbf{b}$ that exceed 2 , so that $k \geq 6$. We can assume that $a_{1}$ is the largest term in $\mathbf{a}$. Since $\mathbf{b} \not \leq \mathbf{a}$, then $a_{1}<k+2$.

We now consider the case where $a_{1}=k$ or $k+1$. It follows that $a_{j} \leq 3$ for $j>1$ and a has altogether at most $n$ terms different from 1 . Hence we can assume that

$$
\mathbf{a}=\left(a_{1}, 2, \ldots, 2,3, \ldots, 3,1, \ldots, 1\right) \in\left[1, s_{2}, s_{3}, n\right] .
$$

If $s_{3}=0$, then Lemma 6.17 gives the desired result. So we assume that $s_{3}>0$. Let

$$
\mathbf{c}=(k-2,2, \ldots, 2,3, \ldots, 3,4,1, \ldots, 1) \in\left[1, s_{2}, s_{3}-1,1, n\right] .
$$

Here $\sum \mathbf{c}=\sum \mathbf{a}-1$ or $\sum \mathbf{a}-2$ and $\mathbf{c} \not \mathbf{a}$, so $\mathbf{c}, \mathbf{a}$ are incomparable. Also, $\mathbf{c} \not \leq \mathbf{b}$ as $\mathbf{b}$ has no term as large as $k-2$. Note that if $s_{3}>1$, then $\mathbf{b}$ has at most one term equal to 3 when $a_{1}=k$ and no such term when $a_{1}=k+1$. Using this, one can show that $\mathbf{b} \not \mathbf{c}$. Thus $\mathbf{c}$ and $\mathbf{b}$ are incomparable. We have that $m(E(\mathbf{a}, \mathbf{c}))=3$ while $m(E(\mathbf{a}, \tau(\mathbf{c})))=1$; so $E(\mathbf{a}, \mathbf{c})$ is definable. To conclude that $E(\mathbf{a}, \mathbf{b})$ is definable, using Lemma 6.7, it remains to show that $E(\mathbf{b}, \mathbf{c})$ is definable. Let

$$
\mathbf{d}=(k+1,2, \ldots, 2,1, \ldots, 1) \in[1, n-1, n] .
$$

We have that $\sum \mathbf{d}>\sum \mathbf{b}$ and $\mathbf{b} \not \leq \mathbf{d}$, so $E(\mathbf{b}, \mathbf{d})$ is definable by Lemma 6.17. Also $\mathbf{c}$ and $\mathbf{d}$ are incomparable, and so $E(\mathbf{c}, \mathbf{d})$ is definable by Lemmas 6.8, 6.14 and 6.17. So it follows that $E(\mathbf{b}, \mathbf{c})$ and $E(\mathbf{a}, \mathbf{b})$ are definable, by Lemma 6.7

It remains to consider the case where $a_{1}<k$. Let $t=\sum \mathbf{a}-(3 n-2) \geq 3$ and $\mathbf{p}=(t, 2, \ldots, 2,1, \ldots, 1) \in[1, n-1, n]$. Now $\sum \mathbf{p}=\sum \mathbf{a}$, so if $\mathbf{a}, \mathbf{p}$ are comparable, then they are equivalent, and then $E(\mathbf{a}, \mathbf{b})$ is definable by Lemma 6.17. We assume that $\mathbf{p}, \mathbf{a}$ are incomparable. It follows that $E(\mathbf{p}, \mathbf{a})$ is definable. Since $\sum \mathbf{p}>\sum \mathbf{b}$, either $\mathbf{p}, \mathbf{b}$ are incomparable, yielding that $E(\mathbf{p}, \mathbf{b})$ is definable by Lemma 6.17 and then $E(\mathbf{a}, \mathbf{b})$ is definable by Lemma 6.7 or else $\mathbf{b}<\mathbf{p}$. So we assume that $\mathbf{b}<\mathbf{p}$. This means that $k+2 \leq t$, or equivalently, $3 n+k \leq \sum \mathbf{a}$.

Now put $\mathbf{q}=(k+1,2, \ldots, 2,1, \ldots, 1) \in[1, n-1, n]$. We have that $\sum \mathbf{q}=$ $3 n+k-1<\sum \mathbf{a}$ and $\mathbf{q} \not \mathbf{a}$ (as a has no term as big as $k$ ). Thus $\mathbf{q}$, a are incomparable. Also a is not equivalent to $(k-1, \ldots, k-1, k, \ldots, k) \in[n, n]$, so $E(\mathbf{a}, \mathbf{q})$ is definable by Lemma 6.14. Also $\mathbf{q}, \mathbf{b}$ are incomparable and $\sum \mathbf{b}<\sum \mathbf{q}$, so $E(\mathbf{q}, \mathbf{b})$ is definable by Lemma 6.17. So it follows that $E(\mathbf{a}, \mathbf{b})$ is definable, by Lemma 6.7. This finishes our proof of Lemma 6.18

Thus, to complete the description of the definable one-based theories, we have to consider the case when 2 occurs in $\mathbf{b}$ less than $n$ times, and there are at least two different indices $i$ and $j$ such that $b_{i}, b_{j}>2$. We note that if $\max \left\{a_{i}\right\} \geq \sum_{b_{i}>2} b_{i}$, then since 1 occurs at most $n$ times in $\mathbf{a}$, the latter would be greater than $\mathbf{b}$. Therefore, $\max \left\{a_{i}\right\} \leq \sum_{b_{i}>2} b_{i}-1$.

Lemma 6.19. If $\max \left\{a_{j}\right\}<\sum_{b_{i}>2} b_{i}-1$, then $E(\mathbf{a}, \mathbf{b})$ is definable. 
Proof. Let $k=\sum_{b_{i}>2} b_{i}-1, t=\sum \mathbf{a}-(3 n-2)$, and $s=\min \{k, t\}$. Take $\mathbf{c}=(1, \ldots, 1,2, \ldots, 2, s) \in[n, n-1,1]$. Then we can argue more or less as above to prove that $E(\mathbf{a}, \mathbf{b})$ is definable.

Thus, for the rest of this section we assume that $\sum \mathbf{a}>\sum \mathbf{b}, \mathbf{a} \geq \mathbf{b}$, the number of terms equal to 2 in $\mathbf{b}$ is less than $n$, there are at least two terms in $\mathbf{b}$ that exceed 2 , and

$$
\max \left\{a_{j}\right\}=a_{1}=\sum_{b_{i}>2} b_{i}-1=k, \text { say. }
$$

Moreover, since 1 occurs at most $n$ times in $\mathbf{a}$ and $\mathbf{a} \geq \mathbf{b}$, then for all $i$ such that $b_{i}>2$ and for all $j>1$ we must have $b_{i}>a_{j}$. From this it follows that $a_{1}>a_{j}$ when $j>1$. Thus if for some $p>0$ there is exactly one term equal to $p$ in $\mathbf{b}$, then $E(\mathbf{a}, \mathbf{b})$ is definable by Lemma 6.5. So, finally, we assume that for each $1 \leq i \leq 2 n$ there is $1 \leq j \leq 2 n, j \neq i$, with $b_{i}=b_{j}$, and we assume that $b=\max \left\{b_{i}\right\}=b_{1}=b_{2}$.

Lemma 6.20. Under all the assumptions above, if in addition there exist $0<i \leq$ $2 n$ such that $2<b_{i}<b-1$, then $E(\mathbf{a}, \mathbf{b})$ is definable.

Proof. Let $i$ be as above. We consider three cases. First, we assume that $i \leq n$. Let $\mathbf{c}$ be obtained from $\mathbf{b}$ by exchanging the first term with $2 b-b_{i}$ and the second term with $b_{i}$. Now $\mathbf{b}, \mathbf{c}$ are incomparable and $\sum \mathbf{c}=\sum \mathbf{b}$, so $E(\mathbf{b}, \mathbf{c})$ is definable. Moreover, for every $j>1, a_{j}<2 b-b_{i}, a_{j}<b_{i}$ and the sum of terms exceeding 2 in $\mathbf{c}$ is the same as the sum of terms exceeding 2 in $\mathbf{b}$, and there is no term in $\mathbf{c}$ as large as $a_{1}$. Hence, a and $\mathbf{c}$ are incomparable, and $E(\mathbf{a}, \mathbf{c})$ is definable by Lemma 6.5 Thus, $E(\mathbf{a}, \mathbf{b})$ is definable by Lemma 6.7.

Next, we assume that there is no term equal to $b-1$ in $\mathbf{b}$. Then take $\mathbf{c}$, which is obtained from $\mathbf{b}$ by exchanging the first term with $b+1$ and the second term with $b-1$, and follow the first case.

Finally, we have to consider the case such that $i>n, b_{j} \in\{1,2, b\}$ for all $0<j \leq n$, and moreover, there exists $\ell>n$ such that $b_{\ell}=b-1$. Take $\mathbf{c}$, which is obtained from $\mathbf{b}$ by exchanging the first term with $2 b-b_{i}-1$ and a term equal to $b-1$ with $b_{i}$, and again follow the first case.

By Lemma 6.20, $b_{i} \in\{1,2, b-1, b\}$. Let us denote the number of those $i \leq 2 n$ such that $b_{i} \in\{b-1, b\}$ by $s$.

Lemma 6.21. With $\mathbf{a}, \mathbf{b}$ as above, $E(\mathbf{a}, \mathbf{b})$ is definable, provided that $s>2$.

Proof. We have either $s=3$ and $b$ occurs three times in $\mathbf{b}$ or $s \geq 4$ and $b$ occurs at least two times in $\mathbf{b}$. Since $b>2$, we have $k \geq 3 b-1$. Let $a_{i}$ be the largest term among $a_{2}, \ldots, a_{2 n}$. Obviously, $a_{i} \geq 2$. Take

$$
\mathbf{c}=\left(k-b, a_{2}, \ldots, a_{i-1}, a_{i}+b-1, a_{i+1}, \ldots, a_{2 n}\right) .
$$

Since $a_{i} \geq 2$, we have $k<(k-b)+\left(a_{i}+b-1\right)$, and therefore $\mathbf{a}$ and $\mathbf{c}$ are incomparable. Moreover, $k-b \geq 3 b-1-b=2 b-1>a_{i}+b-1>a_{i}$. Hence, by Lemma 6.5, $E(\mathbf{a}, \mathbf{c})$ is definable. Observe that the sum of every two terms exceeding 2 in $\mathbf{b}$ is larger than $a_{i}+b-1$ and the sum of every $s-1$ terms exceeding 2 in $\mathbf{b}$ is larger than $k-b$. Moreover, $k-b>b$; therefore, there is no term as large as $k-b$ in $\mathbf{b}$. Hence, $\mathbf{b}$ and $\mathbf{c}$ are incomparable. Now, $k-b<\sum_{b_{i}>2} b_{i}-1$, and by Lemma 6.19, $E(\mathbf{b}, \mathbf{c})$ is definable. Consequently, by Lemma 6.7, $E(\mathbf{a}, \mathbf{b})$ is definable. 
In view of the two lemmas above it remains to consider the last case,

$$
\begin{gathered}
\mathbf{b}=(b, b, 2, \ldots, 2,1, \ldots, 1,) \in[2, n-2, n], \\
\mathbf{a}=\left(2 b-1, a_{2}, \ldots, a_{2 n}\right) .
\end{gathered}
$$

Lemma 6.22. Let $\mathbf{a}, \mathbf{b}$ be as above. If $\mathbf{a}$ is not equivalent to $(2 b-1, b-2, \ldots, b-$ $2, b-1, \ldots, b-1) \in[1, n-1, n]$, then $E(\mathbf{a}, \mathbf{b})$ is definable.

Proof. We have that $a_{j}<b$ for $j>1$. Assume first that $a_{j}<b-1$ for $j>1$ and $a_{i}=\max \left\{a_{1}, \ldots, a_{2 n}\right\}$. Take

$$
\mathbf{c}=\left(2 b-2, a_{2}, \ldots, a_{i-1}, a_{i}+1, a_{i+1}, \ldots, a_{2 n}\right) .
$$

We have that $\mathbf{a}$ and $\mathbf{c}$ are incomparable and $\sum \mathbf{c}=\sum \mathbf{a}$. By Corollary 6.9, $E(\mathbf{a}, \mathbf{c})$ is definable. Since $a_{i}+1<b$, and $2 b-2<2 b-1<\sum_{b_{i}>2} b_{i}$, b and c are incomparable, and $E(\mathbf{b}, \mathbf{c})$ is definable by Lemma 6.19. Hence, by Lemma 6.7. $E(\mathbf{a}, \mathbf{b})$ is definable.

Thus, we can assume that $a_{j}=b-1$ for some $j>1$. First, we assume that $b-1$ occurs in a less than $n$ times. For $w>0$, by $t_{w}$ denote the number of occurrences of $w$ in a. If there is $w, 1 \leq w \leq b-2$, such that $t_{w}-t_{w+1}=q>0$, then define c to be a sequence obtained from a by exchanging occurrences of $w$ and $w+1$, and put $c_{1}=2 b-2$. If the condition does not hold, then there are $i, j>n$ or $1<i, j<n$ such that $a_{i}<a_{j}$. In this case define $\mathbf{c}$ so that $c_{1}=2 b-1+a_{i}-a_{j}$, $c_{i}=a_{j}$, and the remaining terms are as in $\mathbf{a}$. In the second case, $\sum \mathbf{c}=\sum \mathbf{a}$ and $E(\mathbf{a}, \mathbf{c})$ is definable by Corollary 6.9. In the first case, $\sum \mathbf{c} \geq \sum \mathbf{a}$ and $\mathbf{a} \not \leq \mathbf{c}$, and $E(\mathbf{a}, \mathbf{c})$ is definable by Lemma 6.5 $(2 b-2$ is the largest term, occurring exactly once, in this case). Moreover, we know that $a_{1}>c_{1}>b$ and for all $i>1, c_{i}<b$; hence sequences $\mathbf{b}, \mathbf{c}$ are incomparable, and by Lemma 6.19. $E(\mathbf{b}, \mathbf{c})$ is definable. By Lemma 6.7. $E(\mathbf{a}, \mathbf{b})$ is definable (in both cases).

Thus we can assume $t_{b-1}=n$. Then there is $1<i \leq n$ such that $a_{i}<b-2$ (or we are done). Define $\mathbf{c}$ to be the sequence obtained from a by replacing $a_{1}$ by $2 b-2$, and $a_{i}$ by $a_{i}+1$. Observe that $\mathbf{c}$ is incomparable with $\mathbf{a}$ and $\mathbf{b}$. Similarly as above, $E(\mathbf{a}, \mathbf{b})$ is definable in this last case. This completes the proof of Lemma 6.22.

The exceptional case in Lemma 6.22 leads to further new automorphisms of $L(\mathrm{Com})$. Since the situation is completely analogous to that of Lemma 6.15 and Theorem 6.16 we state only the results.

Lemma 6.23. Let either $n>3$ or $n=2, b>2$, $\mathbf{a}=(2 b-1, b-2, \ldots, b-$ $2, b-1, \ldots, b-1) \in[1, n-1, n], \mathbf{b}=(1, \ldots, 1, b, b, 2, \ldots, 2) \in[n, 2, n-2]$, and let $B_{1}=\left(S_{n} \times S_{n}\right)\{\mathbf{a}, \mathbf{b}\}$ and $B_{2}=\left(S_{n} \times S_{n}\right)\left\{\mathbf{a}, \tau_{n}(\mathbf{b})\right\}$. If $E$ is a theory containing exactly one of the blocks $B_{1}$ or $B_{2}$, then the following holds:

(i) All the sequences

$$
\begin{aligned}
& (2 b, 1, \ldots, 1,2, \ldots, 2) \in[1, n, n-2], \\
& (b+1, b, 2, \ldots, 2,1, \ldots, 1) \in[1,1, n-2, n-1], \\
& (b, b, 2, \ldots, 2,1, \ldots, 1) \in[2, n-1, n-2], \\
& (b+2, b, 2, \ldots, 2,1, \ldots, 1) \in[1,1, n-2, n], \text { if } n \geq 4, \\
& (b, b, 3,2, \ldots, 2,1, \ldots, 1) \in[1,1,1, n-3, n-1], \text { if } n \geq 4, \\
& (b, b, 4,2, \ldots, 2,1, \ldots, 1) \in[1,1,1, n-4, n], \text { if } n \geq 4, \\
& (b, b, 2, \ldots, 2,1, \ldots, 1,1) \in[1,1, n-2, n+1], \text { and } \\
& (2 b-1, b-2, \ldots, b-2, b-1, \ldots, b-1,1) \in[1, n-2, n, 1],
\end{aligned}
$$

belong to $J$. 
(ii) There is $i=1$ or 2 such that for every $\mathbf{c}$, if $(\mathbf{a}, \mathbf{c}) \in \pi$, then $\mathbf{c} \in B_{i}$.

(iii) If $f$ is one of the operations $g_{i j}$ or $h_{1}$ and $B$ is a block in $\pi$, then $f(B) \subseteq B_{1}$ implies that $f(B) \subseteq B_{2}$ or $f(B) \subseteq \tau_{n}\left(B_{2}\right)$, and similarly $f(B) \subseteq B_{2}$ implies that $f(B) \subseteq B_{1}$ or $f(B) \subseteq \tau_{n}\left(B_{1}\right)$.

By $\Phi_{b}(E)$ we denote the set of equations obtained from $E$ by exchanging all the equations corresponding to the block $B_{1}$ with all the equations corresponding to the block $B_{2}$. Then we have

Theorem 6.24. For every $t>2$ and every theory $E$, the set $\Phi_{b}(E)$ is a theory, and the mapping $\Phi_{b}$ is an automorphism of $L(C o m)$.

\section{Final RESUlts}

We define the set $\mathcal{E}$ of equations. It is the union of the three classes below, each consisting of two symmetric series:

$$
\begin{aligned}
& e_{n, k}^{1}=((k+2, \underbrace{2, \ldots, 2}_{n-1}, \underbrace{1, \ldots, 1}_{n}),(\underbrace{k+1, \ldots, k+1}_{n-1}, \underbrace{k, \ldots, k}_{n})), \\
& e_{n, k}^{-1}=((k+2,2, \ldots, 2, \underbrace{1, \ldots, 1}_{n}),(\underbrace{k, \ldots, k}_{n}, \underbrace{k+1, \ldots, k+1}_{n})),
\end{aligned}
$$

where $n>0$ and $k>2$ is odd, and moreover $k>3$ for $n=2$ (note that for $n=1$ there are no occurrences of 2 at all);

$$
\begin{aligned}
& f_{n, b}^{1}=((b, b, \underbrace{2, \ldots, 2}_{n-2}, \underbrace{1, \ldots, 1}_{n}),(2 b-1, \underbrace{b-2, \ldots, b-2}_{n-1}, \underbrace{b-1, \ldots, b-1}_{n})), \\
& f_{n, b}^{-1}=((b, b, \underbrace{2, \ldots, 2}_{n-2}, \underbrace{1, \ldots, 1}_{n}),(\underbrace{b-1, \ldots, b-1}_{n}, 2 b-1, \underbrace{b-2, \ldots, b-2}_{n-1})),
\end{aligned}
$$

where either $n>3$ or $n=2$ and $b>2$ (note that for $n=2, b>3$ there are no occurrences of 2 at all);

$$
\begin{aligned}
& g_{k}^{1}=((k+2,1),(k+1, k-1)), \\
& g_{k}^{-1}=((k+2,1),(k-1, k+1)),
\end{aligned}
$$

where $k>3$ is not divisible by 3 .

If $e \in \mathcal{E}$, then by $e^{*}$ we denote the equation obtained from $e$ by exchanging exponents 1 and -1 in the notation above. Using this notation, we can summarize our results in the following theorems.

Theorem 7.1. A theory $E \in L(C o m)$ generated by a single equation $e$ is definable if and only if $e \notin \mathcal{E}$.

Theorem 7.2. If $\Psi$ is an automorphism of $L(C o m)$ and a theory $E$ is onegenerated and not fixed by $\Psi$, then there is an equation $e \in \mathcal{E}$ such that e generates $E$ and $\Psi(E)$ is generated by $e^{*}$.

Let $e \in \mathcal{E}$ and $B_{e}, B_{e}^{*}$ denote the equivalence classes of $e$ and $e^{*}$ (obtained by permuting variables, symmetry, and transitivity). For every theory $E$, by $\Psi_{e}(E)$ denote the set of the equations obtained from $E$ by exchanging the classes $B_{e}$ and $B_{e}^{*}$ (whenever $E$ contains any of these classes).

Theorem 7.3. For every equation $e \in \mathcal{E}$ and every $E \in L(C o m), \Psi_{e}(E) \in$ $L(\mathrm{Com})$, and $\Psi_{e}$ is an automorphism of $L(\mathrm{Com})$.

It is easy to see that for two different equations $e, f \in \mathcal{E}$ automorphisms $\Psi_{e}$ and $\Psi_{f}$, when composed, act independently (on equations consisting of different 
sequences), except for the case $e=e_{1, k}^{s}$ and $f=g_{k}^{t}$, when the sequence ( $k+$ $2,1)$ is involved in both the equations. In this very case, however, if $e_{1, k}^{s}, g_{k}^{t} \in E$ for some $s, t \in\{0,1\}$ and $k>3$ not divisible by 3 , then some equation of the type $((k+1, k-1),(k+1, k))$ with comparable sequences is in $E$, which implies that all the sequences $(k+1, k-1),(k+1, k),(k+2,1)$ are in $J(E)$. It follows that $\Psi_{e}(E)=\Psi_{f}(E)=E$. Therefore not only every finite, but every infinite subset of $\mathcal{E}$ determines an automorphism, and all these automorphisms are different. They obviously form an uncountable Boolean group with the natural operation of composition given by the symmetric difference $\div$ of the sets. Moreover, since every automorphism is determined by its action on the one-based theories, our results show that this is the full group of automorphisms. Denoting by $P(\mathcal{E})$ the power set of $\mathcal{E}$ we have

Theorem 7.4. Every automorphism of the lattice $L(C o m)$ is a composition of a (finite or infinite) set of automorphisms of type $\Psi_{e}$ with $e \in \mathcal{E}$. The group of automorphisms of $L(C o m)$ is a Boolean group isomorphic with $\langle P(\mathcal{E}), \div\rangle$.

Finally we have the following description of definable theories

Theorem 7.5. A theory $E \in L(C o m)$ is definable if and only if $e \in E \cap \mathcal{E}$ implies $e^{*} \in E$.

Since every remainder has a finite character, the problem of definability in $L(C o m)$ is decidable.

\section{ACKNOWLEDGEMENT}

The author would like to thank the anonymous referee for reading the paper very carefully, pointing out many problems, and suggesting many detailed improvements. Also the author thanks Andrzej Kisielewicz for introducing him to this exciting topic and for many fruitful discussions.

\section{REFERENCES}

[1] M. Grech, Irreducible varieties of commutative semigroups, J. Algebra 261 (2003), 207-228. MR1967162 (2004a:20067)

[2] M. Grech, A. Kisielewicz, Covering relation for equational theories of commutative semigroups, J. Algebra 232 (2000), 493-506. MR1792743 (2002g:20102)

[3] J. Ježek, The lattice of equational theories I: Modular elements, Czech. Math. J. 31 (1981), 127-152. MR604120(84e:08007a)

[4] J. Ježek, The lattice of equational theories II: The lattice of full sets of terms, Czech. Math. J. 31 (1982), 127-152. MR604120(84e:08007a)

[5] J. Ježek, The lattice of equational theories III: Definability and automorphisms, Czech. Math. J. 32 (1982), 129-164. MR646718 (84e:08007c)

[6] J. Ježek, The lattice of equational theories IV: Equational theories of finite algebras, Czech. Math. J. 36 (1986), 131-141. MR831318 (87g:08015)

[7] J. Ježek, R. McKenzie, Definability in the lattice of equational theories of semigroups, Semigroup Forum 46 (1993), 199-245. MR.1200214(94a:03052)

[8] A. Kisielewicz, Varieties of commutative semigroups, Trans. Amer. Math. Soc. 342 (1994), 275-306. MR 1211411 (94j:20065)

[9] A. Kisielewicz, Definability in the lattice of equational theories of commutative semigroups, Trans. Amer. Math. Soc. 356 (2004), 3483-3504. MR2055743 (2005a:08011)

[10] A. Kisielewicz, Permutability class of a semigroup, J. Algebra 226 (2000), 295-310. MR 1749890 (2001c:20122)

[11] R. McKenzie, Definability in the lattice of equational theories, Annals of Math. Logic 3 (1971), 197-237. MR0280349(43:6069) 
[12] P. Perkins, Bases for equational theories of semigroups, J. Algebra 11 (1969), 298-314. MR0233911 (38:2232)

[13] A. Tarski, Equational logic and equational theories of algebras, Contributions to Mathematical Logic (Hanover 1966), 275-288, North-Holland, Amsterdam, 1968. MR0237410(38:5692)

Institute of Mathematics, University of WrocŁaW, Pl. Grunwaldzki 2, 50-384 Wroceaw, Poland

E-mail address: Mariusz.Grech@math.uni.wroc.pl 